OPEN ACCESS

Edited by:

Xiumin $\mathrm{Fu}$

South China Botanical Garden, Chinese Academy of Sciences (CAS),

China

Reviewed by:

Mingyue Fu,

Sichuan University, China

Md Abdur Rahim

Sher-e-Bangla Agricultural University,

Bangladesh

*Correspondence:

Zhong Wang

wangzhong19@163.com

Specialty section:

This article was submitted to

Plant Metabolism

and Chemodiversity,

a section of the journal

Frontiers in Plant Science

Received: 20 August 2021 Accepted: 17 November 2021 Published: 15 December 2021

Citation:

Wang N, Shu X, Zhang F Zhuang $W$, Wang $T$ and Wang $Z$ (2021) Comparative Transcriptome

Analysis Identifies Key Regulatory

Genes Involved in Anthocyanin

Metabolism During Flower

Development in Lycoris radiata.

Front. Plant Sci. 12:761862.

doi: $10.3389 / \mathrm{fp} / \mathrm{s} .2021 .761862$

\section{Comparative Transcriptome Analysis Identifies Key Regulatory Genes Involved in Anthocyanin Metabolism During Flower Development in Lycoris radiata}

\author{
Ning Wang 1,2, Xiaochun Shu',2, Fengjiao Zhang 1,2, Weibing Zhuang 1,2, Tao Wang ${ }^{1,2}$ and \\ Zhong Wang 1,2*
}

\footnotetext{
${ }^{1}$ Institute of Botany, Jiangsu Province and Chinese Academy of Sciences, Nanjing, China, ${ }^{2}$ Jiangsu Key Laboratory for the Research and Utilization of Plant Resources, Institute of Botany, Jiangsu Province and Chinese Academy of Sciences, Nanjing, China
}

Lycoris is used as a garden flower due to the colorful and its special flowers. Floral coloration of Lycoris is a vital trait that is mainly regulated via the anthocyanin biosynthetic pathway. In this study, we performed a comparative transcriptome analysis of Lycoris radiata petals at four different flower development stages. A total of 38,798 differentially expressed genes (DEGs) were identified by RNA sequencing, and the correlation between the expression level of the DEGs and the anthocyanin content was explored. The identified DEGs are significantly categorized into 'flavonoid biosynthesis,' 'phenylpropanoid biosynthesis,' 'Tropane, piperidine and pyridine alkaloid biosynthesis,' 'terpenoid backbone biosynthesis' and 'plant hormone signal transduction' by Kyoto Encyclopedia of Genes and Genomes (KEGG) enrichment analysis. The candidate genes involved in anthocyanin accumulation in $L$. radiata petals during flower development stages were also identified, which included 56 structural genes (especially LrDFR1 and LrFLS) as well as 27 key transcription factor DEGs (such as C3H, GATA, $M Y B$, and $N A C$ ). In addition, a key structural gene namely LrDFR1 of anthocyanin biosynthesis pathway was identified as a hub gene in anthocyanin metabolism network. During flower development stages, the expression level of LrDFR1 was positively correlated with the anthocyanin content. Subcellular localization revealed that LrDFR1 is majorly localized in the nucleus, cytoplasm and cell membrane. Overexpression of LrDFR1 increased the anthocyanin accumulation in tobacco leaves and Lycoris petals, suggesting that $L r D F R 1$ acts as a positively regulator of anthocyanin biosynthesis. Our results provide new insights for elucidating the function of anthocyanins in $L$. radiata petal coloring during flower development.

Keywords: Lycoris radiata, transcriptome, anthocyanin, structural genes, phytohormone, transcription factors, dihydroflavonol 4-reductase 


\section{INTRODUCTION}

Plant pigments, such as anthocyanins, carotenoids and chlorophylls, play important roles in affecting the appearance of flower, fruit and seed coloring (Tanaka et al., 2008; Rebecca et al., 2010; Rosas-Saavedra and Stange, 2016; Cui et al., 2021). As an important group of plant pigments, anthocyanins are water soluble and belong to the family of flavonoids. So far, more than 500 different anthocyanins have been isolated from plants (Francavilla and Joye, 2020). They are highly involved in determining flower, seed, fruit and vegetative tissue colors, ranging from pink through scarlet, purple, and blue (Tanaka et al., 2008; Khoo et al., 2017). There are six species of anthocyanins (namely cyanidin, delphinidin, peonidin, malvidin, pelargonidin, and petunidin) in colorful plants (Tanaka et al., 2008; Castaneda-Ovañdo et al., 2009), of which cyanidin is responsible for red-purple coloration, delphinidin contributes to purple or blue-red, and pelargonidin contributes to red and orange (Khoo et al., 2017). Besides, anthocyanins also play various vital functions in plant biological functions, including disease protection, resisting environmental stresses, and promoting pollination (Lev-Yadun and Gould, 2009; Zhang et al., 2014).

Anthocyanins are synthesized in cytosol, and stored in the vacuole. Studies on several plant species, including Arabidopsis (Baudry et al., 2006; Gonzalez et al., 2008; Qi et al., 2011; Xie et al., 2016), agricultural crops (Yang et al., 2019; Dong et al., 2020), fruits (Rahim et al., 2014; Zhou et al., 2015; Jiang et al., 2019; Li C. et al., 2020), vegetable and ornamental plants (Suzuki et al., 2016; Xu et al., 2017; Jin et al., 2018, 2019; Zhu et al., 2019) have revealed that biosynthesis of anthocyanins are controlled by structural and regulatory genes that take part in formation as well as regulation of specific enzymes. The key enzymes including phenylalanine ammonia lyase (PAL), cinnamic acid 4-hydroxylase $(\mathrm{C} 4 \mathrm{H})$, 4-coumarate-CoA ligase (4CL), chalcone synthase (CHS), chalcone isomerase $(\mathrm{CHI})$, flavonone 3-hydroxylase $(\mathrm{F} 3 \mathrm{H})$, dihydroflavonol 4-reductase (DFR), flavonoid 3'-monooxygenase $\left(\mathrm{F} 3^{\prime} \mathrm{H}\right)$, anthocyanin synthase (ANS), as well as UDP-glucoseflavonoid 3-O-glucosyltrasnferase (UFGT) are important in anthocyanin biosynthesis (Koes et al., 2005; Li et al., 2018). Among them, DFR catalyzes the conversion of dihydroflavonols to leucoanthocyanidins, which is one of the final stages of anthocyanin biosynthesis (Shimada et al., 2005; Luo et al., 2016). $D R F$ gene is responsible for plant pigmentation (Lou et al., 2014), and its mutation has been associated with the loss of anthocyanins as well as proanthocyanidins (Liu H. et al., 2019; Jiang et al., 2020; Lim et al., 2020; Feng et al., 2021). Besides, enhancement or activation of DFR gene expression is vital in MYB transcription factor (TF)-based anthocyanin engineering. For example, regulatory roles of MYB TFs in anthocyanin biosynthesis such as Production of Anthocyanin Pigmentation 1 (PAP1, a MYB75 TF), PeMYB2/11/12, PsMYB114L, FtMYBF18, EsMYB90, and FhMYB5 depend on DFR expression (Hsu et al., 2015; Li et al., 2019; Zhang et al., 2019; Dong et al., 2020; Qi et al., 2020). StMYB44 represses anthocyanin accumulation in leaves of tobacco by directly suppressing the activity of the DFR promoter (Liu Y. et al., 2019).
Moreover, some other TFs such as the MYB-bHLH-WD (MBW) complex, B-box, bZIP, MYC, NAC, WRKY, bHLH, MADS-box, and WD could also coordinate anthocyanin biosynthesis initiation by binding to the promoter regions of structural genes (Xu et al., 2015; Zhou et al., 2015; An et al., 2017; Lloyd et al., 2017; Lu et al., 2018; Fang et al., 2019; Jiang et al., 2019). For example, Arabidopsis bHLH TFs (GL3, TT8, and EGL3) and WD40 repeat protein TTG1 regulate anthocyanin biosynthetic gene expressions (Gonzalez et al., 2008; Gerats and Strommer, 2009; Saito et al., 2013). Similarly, anthocyanin biosynthesis in petunia petal cells is controlled by the MBW complex, comprising subgroups of MYB TF (PhAN2 or PhAN4) and bHLH TF (PhAN1 or PhJAF13), as well as the WD40 regulator PhAN11 (Quattrocchio et al., 2006). Strawberry FaMADS1a played a negative role in anthocyanin accumulation via repressing expression of FaPAL6, FaCHS, FaDFR, and FaANS (Lu et al., 2018). Furthermore, apple B-box zinc finger protein MdBBX20 promotes anthocyanin accumulation in response to ultraviolet- $\mathrm{B}$ radiation and low temperature (Fang et al., 2019). DhMYB2 was found to interact with DhbHLH1, thereby regulating anthocyanin secretion in Dendrobium hybrid petals (Li et al., 2017). Therefore, the regulatory mechanisms of TFs on plant color are diverse. The formation of plant flower color is affected by both structural genes and TFs.

The Lycoris species belongs to Amaryllidaceae family, and is a perennial bulb plant native to Northeast Asia, including China, South Korea, and Japan. It consists of about 20 species, of which 15 species and one variety are distributed in China (Zhang et al., 2020). Among them, Lycoris radiata is considered ornamentally and medicinally valuable, as the colorful and special flowers have been used for decoration and the bulbs are notable to produce alkaloids with various biological activities (Park et al., 2019, 2021). Anthocyanins are abundant in Lycoris flowers and also contribute to their color formation (He et al., 2011; Chun et al., 2013; Yue et al., 2019; Park et al., 2021). For example, four critical anthocyanins, namely cyanidin 3-sophoroside, cyanidin 3-xylosylglucoside, cyanidin 3-sambubioside, and pelargonidin 3-xylosylglucoside in $L$. longituba tepals of different colors have been well identified (He et al., 2011). In L radiata flowers, three anthocyanins (cyanidin 3-diglucoside, cyanidin 3-sambubioside, and cyanidin 3-glucoside) were identified (Chun et al., 2013), and their presence during four flower development stages was confirmed more recently (Park et al., 2021). However, the molecular mechanisms of anthocyanins regulating color formation of Lycoris flower remain unclear. Thus, identifying the key genes related to color formation in Lycoris flower would provide a more sufficient genetic resource for manipulation of the related pathways to develop new cultivars with specific flower colors.

In recent years, transcriptome sequencing (RNA-seq) was used as a rapid technique to uncover DEGs, biosynthesis pathways, and TFs related to specific traits in plants (He et al., 2020; Li C. et al., 2020). In this study, we reported the changing profile of anthocyanins and gene expression dynamics in $L$. radiata petals at four developmental stages by integrated analyses of the physiology and transcriptome. We further identified modules with co-expressed genes and candidate hub genes 


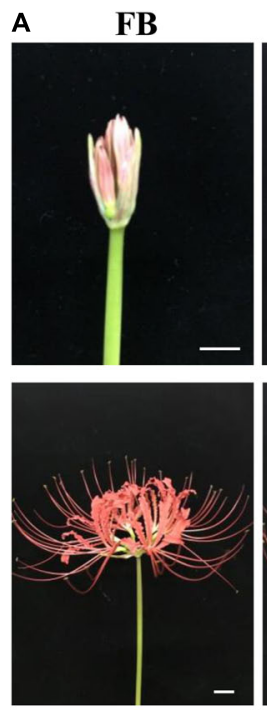

FL2
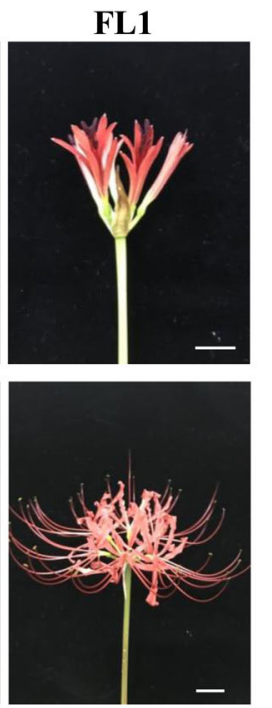

$\mathbf{R}$

FIGURE 1 | Phenotypes and anthocyanins content in petals of $L$. radiata at different development stages. (A) Petals of $L$. radiata at four flower development stages. FB, floral bud stage; FL1, partially opening flower stage; FL2, fully opened flower stage, and R, senescent flower stage. Bars: $1 \mathrm{~cm}$. (B) Anthocyanin levels in L. radiata petals at four flower development stages. Bars with different letters are significantly different at $p<0.05$ according to Duncan's multiple range test.

for anthocyanin accumulation, and revealed $\operatorname{LrDFR} 1$ acts as a positive regulator involved in anthocyanin biosynthesis. Our results may serve as a reference for understanding the regulation of key genes and transcription processes in color formation in the flowers of this esthetically important Lycoris.

\section{MATERIALS AND METHODS}

\section{Plant Materials}

Lycoris radiata (L'Her.) Herb. plants were grown in Experimental Plantation of Institute of Botany, Jiangsu Province and Chinese Academy of Sciences, Nanjing, China. According to the studies reported previously (Yue et al., 2019; Park et al., 2021), three biological replicates of $L$. radiata flowers were sampled at four development stages, which were FB (floral bud stage), FL1 (partially opening flower stage), FL2 (fully opened flower stage) and R (senescent flower stage), as shown in Figure 1A. Each biological replicate was taken from petals of five flowers and pooled together. For gene expression analysis, different $L$. radiata tissues, including scape, stamen, pistil, flower stalk, and petal samples were obtained during flowering time, while leaf, root, as well as bulb samples from the same plants were obtained during the vigorous vegetative growth stage. The fresh samples were harvested and instantly frozen in liquid nitrogen, then kept at $-80^{\circ} \mathrm{C}$ until use.

\section{Measurement of Total Anthocyanins}

Extraction and determination of anthocyanins of $L$. radiata flowers was performed following the protocol of Mehrtens et al. (2005) with minor modifications. Briefly, approximately $0.1 \mathrm{~g}$ fresh petals were ground in $1 \mathrm{~mL}$ of acidic methanol $\left(0.1 \mathrm{~mol} \mathrm{~L}^{-1} \mathrm{HCl}\right)$ and then incubated overnight in the dark at $4^{\circ} \mathrm{C}$ with gentle shaking. After centrifugation for $10 \mathrm{~min}$ at $12,000 \mathrm{rpm}$, the supernatant was diluted four times with acidic methanol and the absorbance was measured at 530 and $657 \mathrm{~nm}$ using a UV-1600 spectrophotometer (SHIMADZU, Kyoto, Japan). The concentration of anthocyanins was calculated using the following formula: $\mathrm{Q}_{\text {Anthocyanins }}=\left(\mathrm{A}_{530}-\right.$ $\left.0.25 \times \mathrm{A}_{657}\right) \times \mathrm{FW}^{-1}$, where $\mathrm{Q}_{\text {Anthocyanins }}$ is the amount of anthocyanins, $A_{530}$ and $A_{657}$ is the absorption at the indicated wavelengths and FW represents the weight of the fresh sample $[\mathrm{g}]$.

\section{Construction of the cDNA Library, Sequencing, and Transcriptome Assembly}

Total RNA was extracted with the mirVana miRNA isolation kit (Thermo Fisher Scientific, Waltham, MA, United States) following the manufacturer's protocol. The quality and quantity of the RNA were examined by the Agilent 2100 Bioanalyzer (Agilent Technologies, Santa Clara, CA, United States). Samples with RNA Integrity Number (RIN) $\geq 7$ were subjected to cDNA library construction using the TruSeq Stranded mRNA LTSample Prep Kit (Illumina, San Diego, CA, United States). Sequencing of the cDNA libraries was done on the Illumina sequencing platform (Illumina HiSeq ${ }^{\mathrm{TM}}$ 2500) by Shanghai OE Biotech. Co. Ltd. (Shanghai, China). Reads were cleaned by removing adapters, as well as low-quality and ambiguous regions, then subjected to de novo assembly using the Trinity software (Grabherr et al., 2011).

\section{Functional Annotation}

Alignment of the assembled unigenes was done against public databases including National Center for Biotechnology Information (NCBI) non-redundant protein $(\mathrm{Nr})$ and nucleotide 
(Nt) database, the Swiss-Prot protein database, Gene Ontology (GO) database, Protein Family (Pfam) database, Kyoto Encyclopedia of Genes and Genomes (KEGG) database, Eukaryotic Ortholog Groups (KOG) database, and eggNOG (evolutionary gene genealogy: Non-supervised Orthologous Groups) database.

\section{Identification of Differentially Expressed Genes}

The expression level of unigenes was calculated using fragments per kilobase per million fragments mapped (FPKM) method (Mortazavi et al., 2008). Identification of DEGs among samples at four development stage was done using the DESeq 2 package implemented in $\mathrm{R}$ software, with cutoff values of $\mid \log 2$ (fold change) $\mid>1$ and $p$-value $<0.05$ algorithms (Young et al., 2010). To visualize the differential expression profiles, we generated a heatmap for the Trimmed Mean of $M$-values (TMM) normalized against FPKM via the pheatmap package in $\mathrm{R}$.

\section{Transcription Factors Analysis}

To predict TFs involved in color formation of $L$. radiata, we utilized the getorf database (mini-size 150) to find the open reading frame (ORF) (Rice et al., 2000) and then used the HMM search database (version 3.0) to align the ORFs to the TF protein domain. The aligned sequences were described according to the TF families available on the PlantTF database version 3.0 (Zhang et al., 2011). Moreover, the Pearson's correlation coefficient (PCC) between these differentially expressed TFs, structure genes and total anthocyanin content of samples was calculated. The TFs with $|\mathrm{PCC}|>0.8$ were selected for subsequent analysis. The TF expression data, which included expression levels for MYB, bHLH, WD40, and the DEGs identified in the flavonoid biosynthetic pathway, was screened using blastx software, with an $e$-value of $1 \mathrm{e}-10$. The target gene sequence was aligned to the protein sequence of the reference species contained in the string database, and the protein interaction relationship of the reference species was used to construct an interaction network. Network visualization for the interaction network related to DFR and DEGs was performed using Cytoscape version 3.6.1.

\section{Gene Cloning and Construction of Expression Vectors}

Cloning of LrDFR1 was based on putative ORFs of unigenes from the RNA-seq database. Primers (Supplementary Table 1) were synthesized for ORF sequence amplification using Tks Gflex ${ }^{\mathrm{TM}}$ DNA Polymerase (Takara, Dalian, China) from L. radiata petal cDNA. Reaction conditions were: $5 \mathrm{~min}$ of $95^{\circ} \mathrm{C}, 35$ cycles for $30 \mathrm{~s}$ at $94^{\circ} \mathrm{C}, 30 \mathrm{~s}$ at $60^{\circ} \mathrm{C}, 1 \mathrm{~min}$ at $72^{\circ} \mathrm{C}$, with extension at $72^{\circ} \mathrm{C}$ for $10 \mathrm{~min}$. PCR products were cloned into pMD19$\mathrm{T}$ simple vectors (Takara, Dalian, China). Afterward, those T-vectors were transferred into $\mathrm{DH} 5 \alpha$ competent cells (Takara, Dalian, China) for amplification. The overexpression vectors of LrDFR1 were established by linking their ORFs into a linear plant transformation vector, pBinGFP4, using the One Step Cloning Kit (Vazyme, Nanjing, China). Then the 35S:LrDFR1 recombinant vectors were transformed into Agrobacterium tumefaciens EHA105 competent cells.

\section{Subcellular Localization and Proanthocyanidin Staining}

The pBinGFP4 vector with LrDFR1-GFP was transformed into the Agrobacterium tumefaciens strain EHA105, and transferred into Nicotiana benthamiana epidermal cells (Sheludko et al., 2007). Cultivation of the transformed N. benthamiana leaves was done for 2-6 days. For co-localization with membranelocalized marker, 35S: PIP2;1-mCherry construction was used (Huang et al., 2019). Assessment of transformed N. benthamiana epidermal cells was observed with confocal laser scanning microscopy (Zeiss LSM780 META, Jena, Germany). For staining of the nuclei, $10 \mathrm{mg} / \mathrm{mL} \mathrm{4}$ 6-diamidino-2-phenylindole (DAPI) was infiltrated into $N$. benthamiana leaves $6 \mathrm{~h}$ before observation.

Staining of proanthocyanidin was conducted as described by An et al. (2015). Briefly, light-treated N. benthamiana leaves were decolorized in a solution of ethanol: glacial acetic acid (3:1). A dimethylaminocinnamaldehyde (DMACA) reagent staining solution (Sigma-Aldrich, St. Louis, MO, United States) was then added for staining.

\section{Agrobacterium-Mediated Transient Transformation System of Lycoris Petals}

The A. tumefaciens harboring 35S:LrDFR1-GFP construct and the control pBinGFP4 vector were prepared for injecting into Lycoris petals, respectively. The recombinant Agrobacterium strains were cultured in YEB broth containing $50 \mu \mathrm{g} \mathrm{mL} \mathrm{mL}^{-1}$ kanamycin and incubated at $28{ }^{\circ} \mathrm{C}$. Then, the collected recombinant Agrobacterium strains were resuspended to $\mathrm{OD}_{600}$ of 0.6 in a buffer with $10 \mathrm{mM}$ 2-(4-Morpholino) ethanesulfonic acid, $10 \mathrm{mM}$ $\mathrm{MgCl}_{2}$, and $120 \mu \mathrm{M}$ acetosyringone. Transformed Lycoris petals were stored for $48 \mathrm{~h}$ in the dark after which they were transferred to a phytotron at a constant photon flux density of $100 \mu \mathrm{mol}$ $\mathrm{m}^{-2} \mathrm{~s}^{-1}$. With 5 days cultivation, Lycoris petals were obtained for anthocyanin level assessment and RNA extraction.

\section{Validation RNA-Seq by Quantitative Real-Time PCR}

For validating gene expression using qRT-PCR, 32 unigenes associated with anthocyanin biosynthesis and phytohormone metabolism were randomly selected (Supplementary Table 2). Total RNA isolation was conducted by using the RNAprep Pure Plant Kit (Tiangen, Beijing, China). First-strand cDNA was synthesized with TransScript One-Step gDNA Removal and cDNA Synthesis SuperMix kit (Takara, Dalian, China), and the extracted RNA was used as template according to manufacturer's instructions. A list of gene-specific primers is provided in Supplementary Table 1 . The quantified expression levels of the tested genes were normalized against the house keeping genes TIP41-like protein (TIP41) according to previous study on L. aurea (Ma et al., 2016). qRT-PCR assays were conducted by the SYBR Premix Ex Taq ${ }^{\mathrm{TM}}$ II kit (Tli RNaseH Plus) (Takara, Dalian, China) in a Bio-Rad iQ5 Gradient RT-PCR system. Reaction conditions were: $30 \mathrm{~s}$ of denaturation at $95^{\circ} \mathrm{C}$ and 40 
amplification cycles (5 s at $95^{\circ} \mathrm{C}, 30 \mathrm{~s}$ at $60^{\circ} \mathrm{C}$ ). Calculation of relative target gene expression levels was done using the $2^{-\Delta \Delta C t}$ method (Livak and Schmittgen, 2001). Experiments were conducted using three independent biological and three technical replicates.

\section{Statistical Analysis}

Statistical analyses were done by SPSS version 10.0 software (IBM Corporation, Armonk, NY, United States). The significant difference among sets of data was determined by one-way analysis of variance (ANOVA) with Duncan's multiple range test $(p<0.05)$ or a significant $t$-test $\left({ }^{* *} p<0.01,{ }^{*} p<0.05\right)$. All the results are presented as the mean \pm standard deviation (SD).

\section{RESULTS}

\section{Anthocyanin Levels in Lycoris radiata Petal During Flower Development Stages}

During the red flower development of $L$. radiata, petals underwent a rapid color change from slight red to brilliant red (Figure 1A). At the flower bud (FB) stage, a slight red color was observed, then the color intensity was significantly increased with rapid elongation of petals in FL1. Subsequently, the intensity of L. radiata decreased at FL2 and R stages (Figure 1A). We thus investigated the changes of anthocyanin contents in $L$. radiata at four different petal development stages. Notably, anthocyanin content at FL1 stage was significantly higher than that of FB, FL2 and R stages (Figure 1B), suggesting that changes in anthocyanin levels could be the main reason for red color formation of L. radiata.

\section{Transcriptome Sequencing and de novo Assembly}

To further study the molecular mechanism of $L$. radiata petal coloring during flower development, twelve libraries were established using samples at four flower development (FB, FL1, FL2, and R) stages (three biological replicates for samples at each development stage), and a total of 644.93 million raw reads as well as $96.73 \mathrm{~Gb}$ raw bases were obtained. After eliminating the adaptor, poor-quality sequences, and ambiguous reads, 634.09 million clean reads and $89.86 \mathrm{~Gb}$ clean bases were retrieved from 12 samples (Supplementary Table 3 ). The quality score above 30 (Q30) of each library was 93.75-94.91\%, and GC percentages ranged from 44.99-46.51\% (Supplementary Table 3). By using Trinity software, the de novo assembly of 12 petal transcriptomes totally generated 87,584 unigenes with an average length of 942 bp (Supplementary Table 4). Sequence length distribution showed that 27,073 (30.91\%) unigenes had a mean length $\geq 1000$ bp (Supplementary Figure 1 and Supplementary Table 4). The N50 was determined to be $1,334 \mathrm{bp}$, which indicated that the quality of sequence assembly was good.

FPKM values were used to estimate the transcription levels of unigenes. More than $50.0 \%$ of unigenes had FPKM values above 1 (Supplementary Figure 2). In addition, the use of relative unigene expression obtained from FPKM for principal component analysis (PCA) showed $52.10 \%$ variability among the samples (Supplementary Figure 3). Moreover, heatmap coefficient matrix analysis of the samples based on the FPKM values showed that most biological replicates (except FB3 sample) exhibited similar expression patterns, indicating relatively high reliability of our sequencing data (Supplementary Figure 4).

\section{Functional Annotations and Unigene Classifications}

All of the unigenes were annotated by BLAST search against the public databases. The results revealed that $40,974(46.78 \%)$, 29,476 (33.65\%), 37,487 (42.8\%), and 22,318 (25.48\%) unigenes were annotated to the Nr, Swiss-Prot, eggNOG, and Pfam databases, respectively. Taken the entire public databases together, a total of $41,534(47.42 \%)$ unigenes could be successfully annotated (Supplementary Table 5). To elucidate their main biological functions, GO, KOG, and KEGG pathway assessments were also performed (Supplementary Figures 57). Consequently, 27,296 (31.17\%) unigenes were assigned into three main categories including "biological process" (BP), "cellular component" (CC), and "molecular function (MF)," which could be further distributed under 50 GO terms (Supplementary Figure 5). In addition. 15,122 (17.27\%) unigenes were associated with 126 KEGG pathways, and category 'Metabolism' (6187 unigenes) was the most abundant (Supplementary Figure 6). Moreover, the KOG analysis showed that 23,858 (27.24\%) annotated unigenes were assigned into 25 classes (Supplementary Figure 7).

\section{Identification of Differentially Expressed Genes in Lycoris radiata Petal During Flower Development Stages}

To identify the key DEGs involved in L. radiata petal color transitions, six pair-wise comparison groups (FL1 vs. R, FB vs. $\mathrm{R}, \mathrm{FL} 2$ vs. $\mathrm{R}, \mathrm{FL} 2$ vs. FL1, FL1 vs. FB, and FL2 vs. FB) were conducted (Figure 2). A total of 38,798 DEGs were identified among all samples based on a $\mid \log _{2}$ fold change $\mid>1$ at $p<0.05$. Among these comparison groups, the largest abundance of DEGs $(23,202)$ was found between FB and R libraries, of which 10,958 and 12,244 genes were down-regulated and upregulated, respectively (Figure $\mathbf{2 A}$ ). Conversely, the smallest abundance of DEGs $(9,057)$ was recorded between FL2 and FL1 libraries, with 5,033 and 4,024 of them down-regulated and up-regulated, respectively (Figure 2A). Furthermore, the overlap DEGs among the six comparison groups were screened. The results indicated that 38 genes were differentially expressed among all the comparisons, which indicated that these DEGs might have key functions in the color expression of different petals (Figure 2B and Supplementary Table 6).

\section{Functional Annotation of Differentially Expressed Genes}

To elaborate the functions of DEGs and identify genes involved in regulating anthocyanin accumulation in L. radiata, all the DEGs were firstly subjected to GO analyses, and 14,555 of the 38,798 DEGs were assigned to GO annotations (Supplementary Table 7 

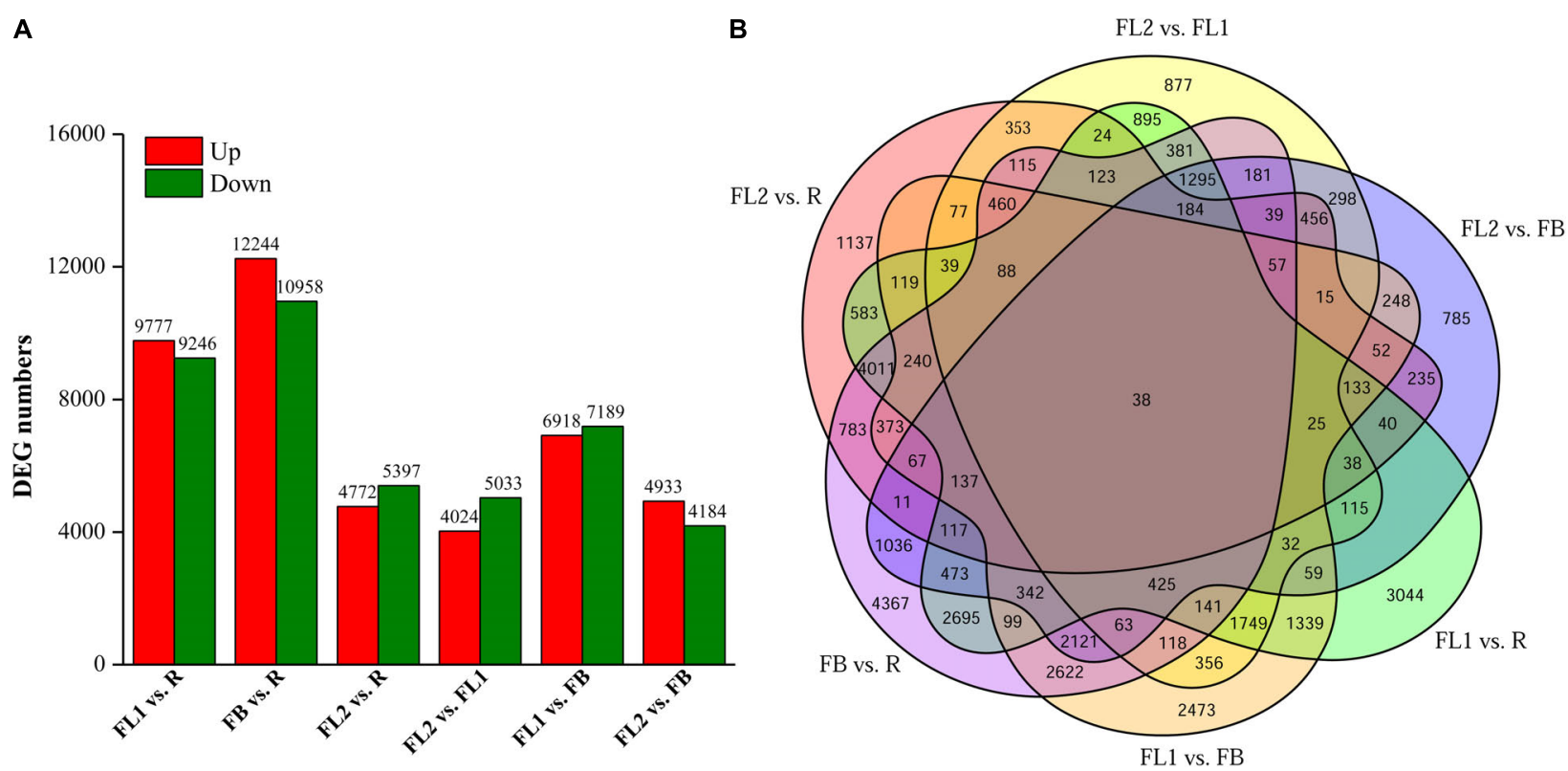

FIGURE 2 | Statistics of differentially expressed genes (DEGs) between two different samples at flower development stages. (A) Numbers of DEGs in various pair-wise comparisons. (B) Venn diagram for the numbers of DEGs as shown by pair-wise comparisons. FB, floral bud stage; FL1, partially opening flower stage; FL2, fully opened flower stage, and R, senescent flower stage.

and Figure 3A). In the biological process category, most of the DEGs were mapped to 'cellular process' (9,476, 20.23\%), 'metabolic process' $(8,073,17.23 \%)$, and 'response to stimulus' $(4,181,8.92 \%)$ terms. In the cellular component category, more than $63.08 \%$ of DEGs were enriched in 'cell', 'cell part' and 'organelle' terms, but for molecular function, nearly $86.13 \%$ of DEGs were mapped to 'catalytic activity' and 'binding' terms (Figure 3A). For the KEGG annotation results, 7,631 DEGs among all samples were also mapped to 126 KEGG pathways (Supplementary Table 7). Comparisons across the samples at four petal development stages revealed significant enrichment of DEGs in 'flavonoid biosynthesis,' 'phenylpropanoid biosynthesis', 'Tropane, piperidine and pyridine alkaloid biosynthesis,' 'terpenoid backbone biosynthesis' as well as 'plant hormone signal transduction' pathways (Figure 3B and Supplementary Figure 8). For example, the significantly enriched KEGG pathway term 'Tropane, piperidine and pyridine alkaloid biosynthesis' was shared in all the comparisons. The 'flavonoid biosynthesis' pathway was enriched in FL1 vs. R, FB vs. R, FL2 vs. R, FL2 vs. FL1, and FL2 vs. FB, but not in FL1 vs. FB. In addition, the 'plant hormone signal transduction' pathway was enriched in FB vs. R, FL2 vs. FB, and FL2 vs. FL1 (Supplementary Figure 8).

\section{Identification of Key Differentially Expressed Genes Responsible for the Anthocyanin Biosynthesis Pathway}

To elucidate the molecular basis underlying difference in anthocyanin biosynthesis among the four flower development stages in $L$. radiata, DEGs involved in the anthocyanin synthesis pathway were identified. The results revealed that 56
DEGs were enriched in the anthocyanin synthesis pathway, including $P A L, C 4 H, 4 C L, C H S, C H I, F 3 H, F 3^{\prime} H, D F R$, ANS, UFGT, FLS, ANR, and LAR (Figure 4A). Moreover, the Pearson's correlation coefficient between the expression level of these DEGs and the total anthocyanins content was further calculated (Figure 4B). The results showed that 23 DEGs negatively regulated anthocyanin synthesis, whereas 33 DEGs positively regulated the anthocyanin synthesis. Among them, the expression level of two DEGs, namely LrDFR1 (DN43960) and LrDFR2 (DN42380) indicated a significant positive correlation with the total anthocyanins content in petals during the flower development stages, while LrFLS (DN37334) indicated a significant negative correlation with the total anthocyanins content ( $|\mathrm{PCC}|>0.8$, Figure 4B and Table 1$)$, suggesting that these three DEGs may have an essential role in anthocyanin accumulation.

\section{Identification of Transcription Factors Related to Anthocyanin Biosynthesis in the Petals of Lycoris radiata}

Transcription factors were subsequently predicted to whether modulate anthocyanin accumulation and biosynthesis in L. radiata petals during flowering development stages. In this study, a total of 1,631 TFs were identified by searching the TF database. The classified results indicated that most of these TFs belonged to the MYB, C2C2, AP2/ERF, C2H2, and bHLH family (Supplementary Figure 9). Furthermore, the differentially expressed TFs (721) were characterized by analyzing their FPKM values (Supplementary Figure 9). Importantly, coexpression modules of these 721 TF DEGs were analyzed with 


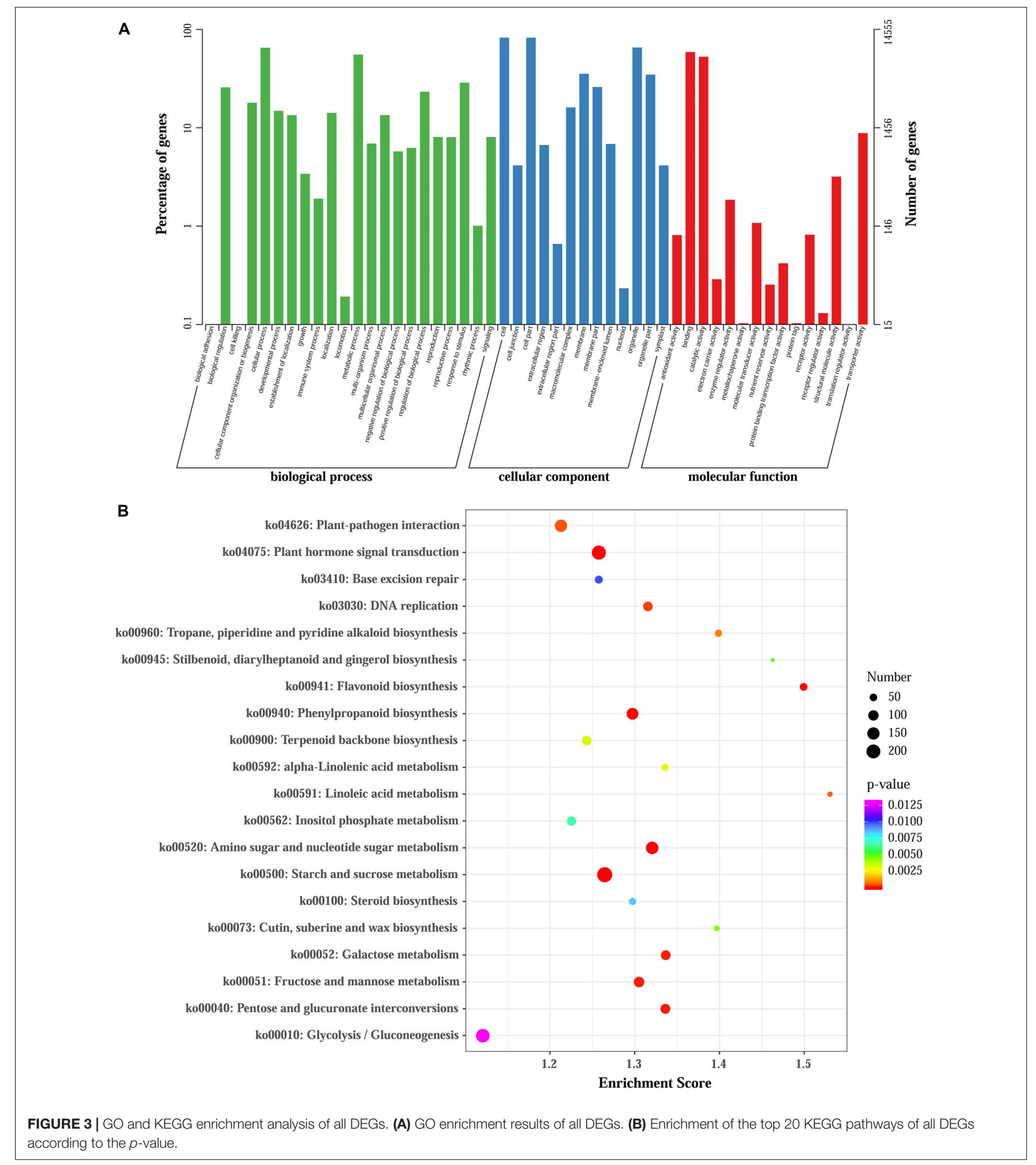

Short Time-series Expression Miner (STEM) software. In all, six clusters of 272 TF DEGs were detected (Figure 5A and Supplementary Table 8).

On the other hand, by calculating the PCC between the expression level of 721 TF DEGs and the total anthocyanins content, 27 TFs genes ( $\mathrm{PCC} \mid>0.8)$ involved in the accumulation of anthocyanins were identified, including 10 positive regulators and 17 negative regulators (Table 1). These 10 positive regulators, including MYB (1), AP2/ERF (1), bHLH (1), bZIP (1), NAC (1), NF-X1 (1), and Trihelixs (4) genes, 

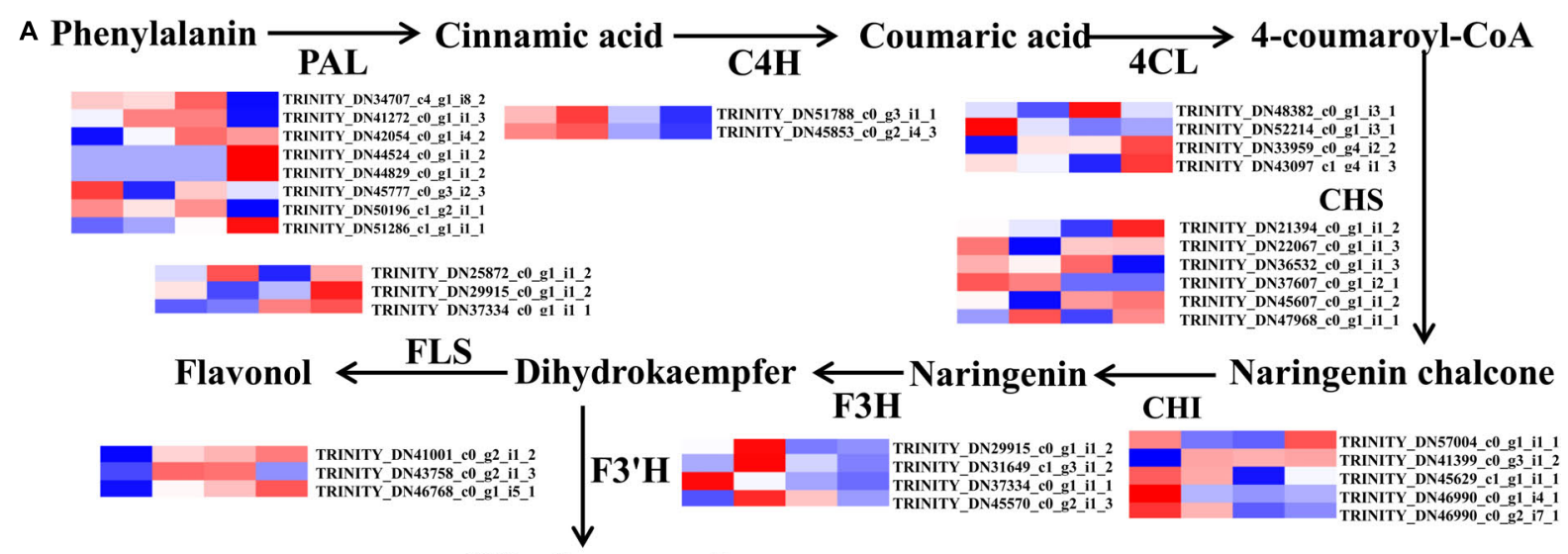

Dihydroquerceti

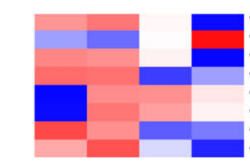

TRINITY_DN49637_c0_g1_i16_1 TRINITY DN49637 C0 $\mathrm{g} 2$ i1 TRINITY DN39015 C0 g1 i1 3 TRINITY_DN41815_c0_g1_i8_3 TRINITY_DN42380_C0_g4_i2 _2 TRINITY_DN43960COg1_i7 3 TRINIT_DN44086_c0_gl_i12

Leucoanthocyanidins

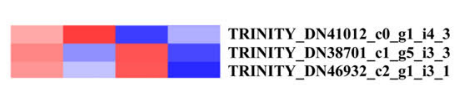

DFR

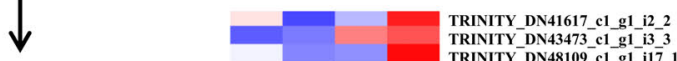
TRINITY_DN43473_c1_g1_i33
TRINITY_DN48109_cl_g1_i1
TRINITY_DN51829_c0_g1_i1_1 TRINITY<smiles>CCC[SiH3]</smiles>

Anthocyanidin

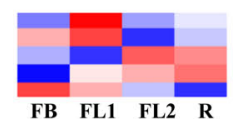
TRINITY_DN43215_c0_g2_i1_3
TRINITY_DN38114_c0_g1_i7_-2
TRINITY_DN3855_c0_g1_i2_-3
TRINITY_DN4465_c0_g2 i2_1
TRINITY_DN48727c0_g1 i1_1

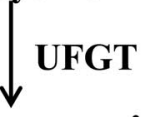

Anthocyanins

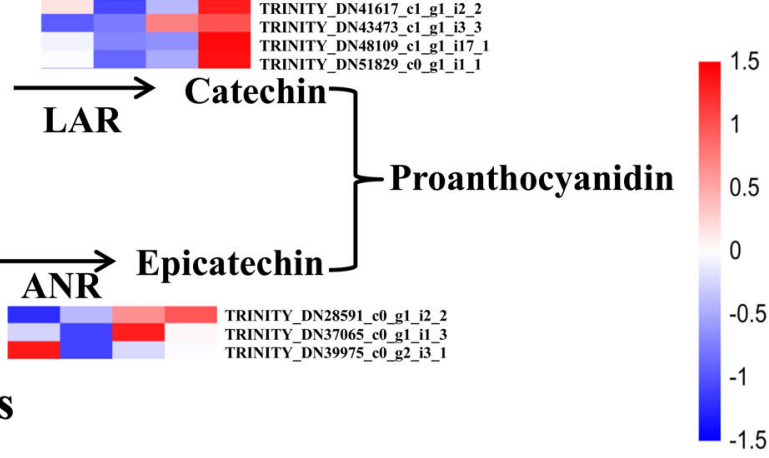

B

\begin{tabular}{|c|c|c|c|c|c|c|}
\hline $\begin{array}{l}\text { DN34707 } \\
\text { PAL } \\
\text { 0.04 }\end{array}$ & $\begin{array}{c}\text { DN51788 } \\
\text { C4H } \\
0.21\end{array}$ & $\begin{array}{c}\text { DN36532 } \\
\text { CHS } \\
0.35\end{array}$ & $\begin{array}{c}\text { DN46990 } \\
\text { CHI } \\
-0.44\end{array}$ & $\begin{array}{c}\text { DN49637 } \\
\text { DFR } \\
0.05\end{array}$ & $\begin{array}{c}\text { DN41012 } \\
\text { ANS } \\
0.14\end{array}$ & $\begin{array}{c}\text { DN25872 } \\
\text { FLS } \\
\mathbf{0 . 3 2}\end{array}$ \\
\hline $\begin{array}{c}\text { DN41272 } \\
\text { PAL } \\
0.36\end{array}$ & $\begin{array}{c}\text { DN45853 } \\
\text { C4H } \\
\text { 0.04 }\end{array}$ & $\begin{array}{c}\text { DN37607 } \\
\text { CHS } \\
-\mathbf{0 . 2 7}\end{array}$ & $\begin{array}{c}\text { DN29915 } \\
\text { F3H } \\
\mathbf{0 . 5 6}\end{array}$ & $\begin{array}{c}\text { DN49637 } \\
\text { DFR } \\
\text { 0.02 }\end{array}$ & $\begin{array}{c}\text { DN38701 } \\
\text { ANS } \\
-0.25\end{array}$ & $\begin{array}{c}\text { DN41617 } \\
\text { FLS } \\
-\mathbf{0 . 2 2}\end{array}$ \\
\hline $\begin{array}{l}\text { DN42054 } \\
\text { PAL } \\
0.32\end{array}$ & $\begin{array}{c}\text { DN48382 } \\
4 C L \\
0.01\end{array}$ & $\begin{array}{c}\text { DN45607 } \\
\text { CHS } \\
-0.06\end{array}$ & $\begin{array}{c}\text { DN31649 } \\
\text { F3H } \\
0.66\end{array}$ & $\begin{array}{c}\text { DN39015 } \\
\text { DFR } \\
-\mathbf{0 . 0 4}\end{array}$ & $\begin{array}{c}\text { DN46932 } \\
\text { ANS } \\
-0.21\end{array}$ & $\begin{array}{c}\text { DN43473 } \\
\text { LAR } \\
0.10\end{array}$ \\
\hline $\begin{array}{l}\text { DN44524 } \\
\text { PAL } \\
0.32\end{array}$ & $\begin{array}{c}\text { DN52214 } \\
4 C L \\
-0.66\end{array}$ & $\begin{array}{c}\text { DN47968 } \\
\text { CHS } \\
0.45\end{array}$ & $\begin{array}{c}\text { DN37334 } \\
\text { FLS } \\
-0.81\end{array}$ & $\begin{array}{c}\text { DN41815 } \\
\text { DFR } \\
-0.14\end{array}$ & $\begin{array}{c}\text { DN43215 } \\
\text { UFGT } \\
0.31\end{array}$ & $\begin{array}{c}\text { DN48109 } \\
\text { LAR } \\
-0.15\end{array}$ \\
\hline $\begin{array}{l}\text { DN44829 } \\
\text { PAL } \\
0.01\end{array}$ & $\begin{array}{l}\text { DN33959 } \\
\text { 4CL } \\
0.52\end{array}$ & $\begin{array}{c}\text { DN57004 } \\
\text { CHI } \\
-0.52\end{array}$ & $\begin{array}{c}\text { DN45570 } \\
\text { F3H } \\
0.61\end{array}$ & $\begin{array}{c}\text { DN42380 } \\
\text { DFR } \\
0.80\end{array}$ & $\underset{\substack{\text { UFGT } \\
\text { O.17 }}}{\text { DN8114 }}$ & $\begin{array}{l}\text { DN51829 } \\
\text { LAR } \\
\text { 0.01 }\end{array}$ \\
\hline $\begin{array}{c}\text { DN45777 } \\
\text { PAL } \\
-0.47\end{array}$ & $\begin{array}{c}\text { DN43097 } \\
\text { 4CL } \\
-0.17\end{array}$ & $\begin{array}{l}\text { DN41399 } \\
\text { CHI } \\
0.42\end{array}$ & $\begin{array}{c}\text { DN41001 } \\
\text { F3'H } \\
0.25\end{array}$ & $\begin{array}{l}\text { DN43960 } \\
\text { DFR } \\
0.86\end{array}$ & $\begin{array}{c}\text { DN38555 } \\
\text { UFGT } \\
0.14\end{array}$ & $\begin{array}{c}\text { DN28591 } \\
\text { ANR } \\
0.13\end{array}$ \\
\hline $\begin{array}{c}\text { DN50196 } \\
\text { PAL } \\
-0.20\end{array}$ & $\begin{array}{c}\text { DN21394 } \\
\text { CHS } \\
-0.07\end{array}$ & $\begin{array}{c}\text { DN45629 } \\
\text { CHI } \\
-0.39\end{array}$ & $\begin{array}{c}\text { DN43758 } \\
\text { F3'H } \\
0.63\end{array}$ & $\begin{array}{c}\text { DN44086 } \\
\text { DFR } \\
-0.39\end{array}$ & $\begin{array}{c}\text { DN44965 } \\
\text { UFGT } \\
0.51\end{array}$ & $\begin{array}{c}\text { DN37065 } \\
\text { ANR } \\
0.03\end{array}$ \\
\hline $\begin{array}{l}\text { DN51286 } \\
\text { PAL } \\
0.12\end{array}$ & $\begin{array}{c}\text { DN22067 } \\
\text { CHS } \\
-\mathbf{0 . 3 0}\end{array}$ & $\begin{array}{c}\text { DN46990 } \\
\text { CHI } \\
-0.64\end{array}$ & $\begin{array}{c}\text { DN46768 } \\
\text { F3'H } \\
\mathbf{0 . 4 5}\end{array}$ & $\begin{array}{c}\text { DN45181 } \\
\text { DFR } \\
0.14\end{array}$ & $\begin{array}{c}\text { DN48727 } \\
\text { UFGT } \\
-0.54\end{array}$ & $\begin{array}{c}\text { DN39975 } \\
\text { ANR } \\
-0.64\end{array}$ \\
\hline
\end{tabular}

FIGURE 4 | Analysis of DEGs involved in anthocyanin biosynthesis pathway in L. radiata. (A) Anthocyanin biosynthesis pathway and the log 2 transformed FPKM values of DEGs associated with structural enzyme genes were used to draw the heatmap. The enzymes include 4-coumarateCoA ligase (4CL), phenylalanine ammonia lyase (PAL), chalcone synthase (CHS), flavone 3-hydroxylase $(\mathrm{F} 3 \mathrm{H})$, chalcone isomerase $(\mathrm{CHI})$, flavonoid $3^{\prime}$-hydroxylase $\left(\mathrm{F} 3^{\prime} \mathrm{H}\right)$, dihydroflavonol reductase (DFR), flavonol synthase (FLS), UDP-flavonoid glucosyl transferase (UFGT), anthocyanidin reductase (ANR), and leucoanthocyanidin reductase (LAR). FB, floral bud stage; FL1, partially opening flower stage; FL2, fully opened flower stage, and R, senescent flower stage. Color gradients comprise red, white, and blue, representing genes that were upregulated, not regulated, as well as downregulated, respectively. (B) The heatmap analysis of all DEGs in anthocyanin biosynthesis pathway according to the FPKM value.

likely act to improve anthocyanin synthesis during $L$. radiata petal development stages. However, the 17 negative regulators, including Alfin-like (1), AP2/ERF (1), GATA (1), GRFs (2), bHLHs
(2), MYBs (2), C2H2s (2), C3Hs (4), MADS (1), and NAC (1), might act as repressors in $L$. radiata anthocyanin biosynthesis (Table 1). Notably, 11 of 17 negative TF regulators (subclass 4, 
TABLE 1 | The candidate TFs and the key structural gene involved in anthocyanin accumulation.

\begin{tabular}{|c|c|c|c|c|}
\hline Gene family & Gene ID & Annotation & Correlation with total anthocyanin & $p$-value \\
\hline FLS & TRINITY_DN37334_c0_g1_i1_1 & Flavonol synthase/flavanone 3-hydroxylase-like & -0.8058 & 0.0015 \\
\hline \multirow[t]{2}{*}{$D F R$} & TRINITY_DN42380_c0_g4_i2_2 & Dihydroflavonol 4-reductase LrDFR2 & 0.8045 & 0.0016 \\
\hline & TRINITY_DN43960_c0_g1_i7_3 & Dihydroflavonol 4-reductase (LrDFR1) & 0.8655 & 0.0002 \\
\hline Alfin-like & TRINITY_DN45802_c0_g1_i2_1 & PHD finger protein ALFIN-LIKE 6-like & -0.9302 & 1.16E-05 \\
\hline \multirow[t]{2}{*}{ AP2/ERF } & TRINITY_DN42881_c0_g1_i1_3 & AP2 domain-containing transcription factor 2 & 0.8584 & 0.0003 \\
\hline & TRINITY_DN13573_c0_g1_i1_1 & AP2 domain-containing transcription factor 2 & -0.8304 & 0.0008 \\
\hline \multirow[t]{3}{*}{ bHLH } & TRINITY_DN36174_c0_g1_i1_1 & Transcription factor bHLH30-like & -0.8631 & 0.0002 \\
\hline & TRINITY_DN41224_c0_g1_i1_1 & Transcription factor bHLH57-like & -0.8494 & 0.0004 \\
\hline & TRINITY_DN48856_c0_g1_i6_1 & Transcription factor bHLH48-like & 0.8305 & 0.0008 \\
\hline bZIP & TRINITY_DN27549_c0_g1_i3_2 & bZIP transcription factor 11-like & 0.8317 & 0.0007 \\
\hline GATA & TRINITY_DN30983_c0_g2_i1_2 & GATA transcription factor 3-like isoform X2 & -0.8175 & 0.0011 \\
\hline \multirow[t]{2}{*}{ GRF } & TRINITY_DN37111_c0_g1_i4_2 & Growth-regulating factor 4-like & -0.8349 & 0.0007 \\
\hline & TRINITY_DN48073_c0_g1_i1_1 & Growth-regulating factor 7 -like & -0.8084 & 0.0014 \\
\hline \multirow[t]{2}{*}{$\mathrm{C} 2 \mathrm{H} 2$} & TRINITY_DN44933_c0_g1_i2_1 & Histone deacetylase HDT2-like & -0.8566 & 0.0003 \\
\hline & TRINITY_DN48967_c0_g1_i23_1 & Histone deacetylase HDT2 & -0.8434 & 0.0005 \\
\hline \multirow[t]{4}{*}{$\mathrm{C} 3 \mathrm{H}$} & TRINITY_DN36142_c1_g2_i3_3 & Zinc finger $\mathrm{CCCH}$ domain-containing protein 8 & -0.8517 & 0.0004 \\
\hline & TRINITY_DN42291_c0_g1_i1_1 & Zinc finger $\mathrm{CCCH}$ domain-containing protein 59 & -0.8752 & 0.0001 \\
\hline & TRINITY_DN60484_c0_g1_i1_1 & Zinc finger $\mathrm{CCCH}$ domain-containing protein 44 & -0.8130 & 0.0013 \\
\hline & TRINITY_DN48680_c0_g1_i6_1 & Zinc finger $\mathrm{CCCH}$ domain-containing protein 8 & -0.8535 & 0.0004 \\
\hline MADS & TRINITY_DN50153_c1_g1_i10_1 & Transcription factor, MADS-box & -0.8239 & 0.0009 \\
\hline \multirow[t]{3}{*}{ MYB } & TRINITY_DN33872_c0_g1_i1_3 & MYB transcription factor & 0.8101 & 0.0013 \\
\hline & TRINITY_DN45447_c0_g1_i7_1 & MYB transcription factor & -0.8298 & 0.0008 \\
\hline & TRINITY_DN51496_c0_g1_i9_1 & MYB transcription factor & -0.8245 & 0.0009 \\
\hline \multirow[t]{2}{*}{ NAC } & TRINITY_DN39353_c0_g2_i1_1 & NAC domain-containing protein 43 & -0.8351 & 0.0007 \\
\hline & TRINITY_DN39797_c0_g2_i6_3 & NAC domain-containing protein 17-like & 0.8215 & 0.0010 \\
\hline$N F-X 1$ & TRINITY_DN33064_c0_g1_i1_2 & NF-X1-type zinc finger protein NFXL2 & 0.8369 & 0.0006 \\
\hline \multirow[t]{4}{*}{ Trihelix } & TRINITY_DN39907_c1_g1_i5_1 & Trihelix transcription factor GTL1 & 0.8138 & 0.0012 \\
\hline & TRINITY_DN41155_c0_g2_i2_2 & Trihelix transcription factor GTL1-like & 0.8208 & 0.0010 \\
\hline & TRINITY_DN43481_c0_g2_i1_1 & Trihelix transcription factor ASIL2-like & 0.8571 & 0.0003 \\
\hline & TRINITY_DN45677_c0_g1_i7_3 & Trihelix transcription factor GTL1 & 0.8042 & 0.0016 \\
\hline
\end{tabular}

5 , and 6) and one positive regulator (subclass 2) were enriched in TF co-expression modules (Supplementary Table 8).

Previous studies have reported that bHLH, MYB and WD40 TFs regulate anthocyanin biosynthesis thereby activating or repressing transcription of anthocyanin structural genes. We then performed unigenes regarding to MYB, bHLH and WD40, as well as 56 DEGs involved in anthocyanin biosynthesis (Figure 5) to analyze their interaction network and hope to identify the hub TF genes that could affect anthocyanin biosynthesis pathway. The results showed that four DFRs, four $M Y B s$, two WD40s, two 4CLs, one $F 3^{\prime} H$, one UFGT, one CHS, one ANS, one FLS, and one CHI were selected as hub genes based on their connection position in the network modules, expression pattern and functional annotation (Supplementary Table 9a and Supplementary Figure 10). Furthermore, those genes (shown in Supplementary Table 9a) and 27 key TF genes (Table 1) were selected to build the interaction network for further analysis. Among them, LrDFR1 (DN43960) and LrFLS (DN37334) could be regarded as key hub genes for participating anthocyanin biosynthesis. Two MYBs (DN45447 and DN33872), two NACs (DN39353 and DN39797), one C3H (DN42291), and one GATA (DN30983) TF genes were identified as hub genes in regulating anthocyanin biosynthesis (Figure 5B and Supplementary Table 9b). The above results indicate that these eight genes may play essential roles in anthocyanin synthesis in L. radiata during petal development.

\section{Validation of RNA-seq Data by qRT-PCR}

To validate the accuracy and transcription profiles revealed by the RNA-seq data, 32 unigenes were selected for qRT-PCR assays. The relative expression levels of these 32 genes were normalized to the expression of LrTIP41, and compared with the RNA-Seq data, as shown in Figure 6A. Further linear regression analysis revealed that the expression levels of these genes were well correlated with the RNA-Seq results (Figure 6B, $R^{2}>0.76$ ), indicating that the RNA-seq data were credible and accurate.

\section{LrDFR1 Is Involved in Anthocyanin Biosynthesis in Lycoris radiata}

In this study, we cloned LrDFR1 gene (DN43960) from L. radiata. The full-length cDNA of LrDFR1 is 1113 bp in length and it encodes a 370 amino acid protein with a molecular weight of $41.67 \mathrm{kDa}$ (Supplementary Table 10). 

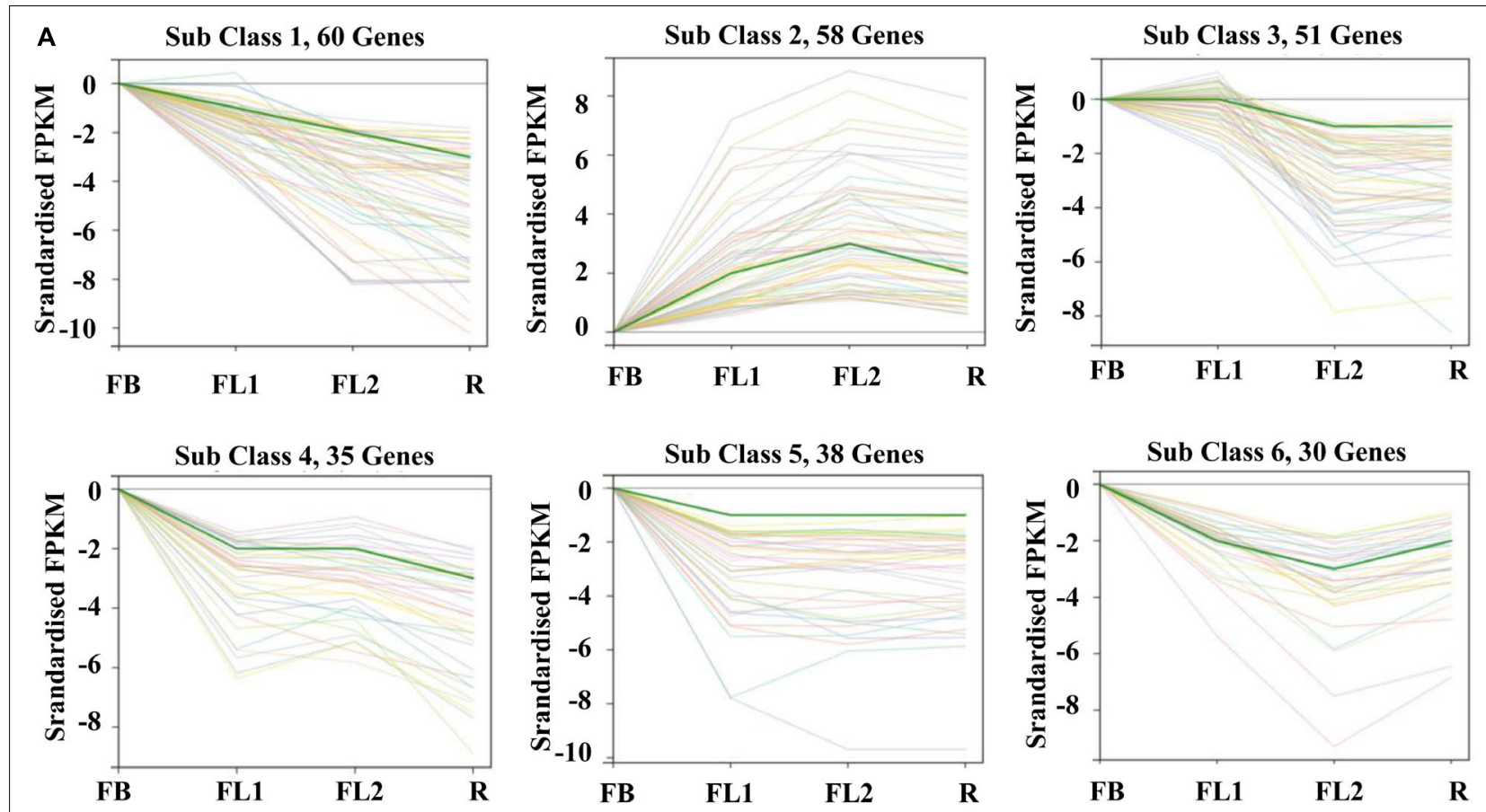

B

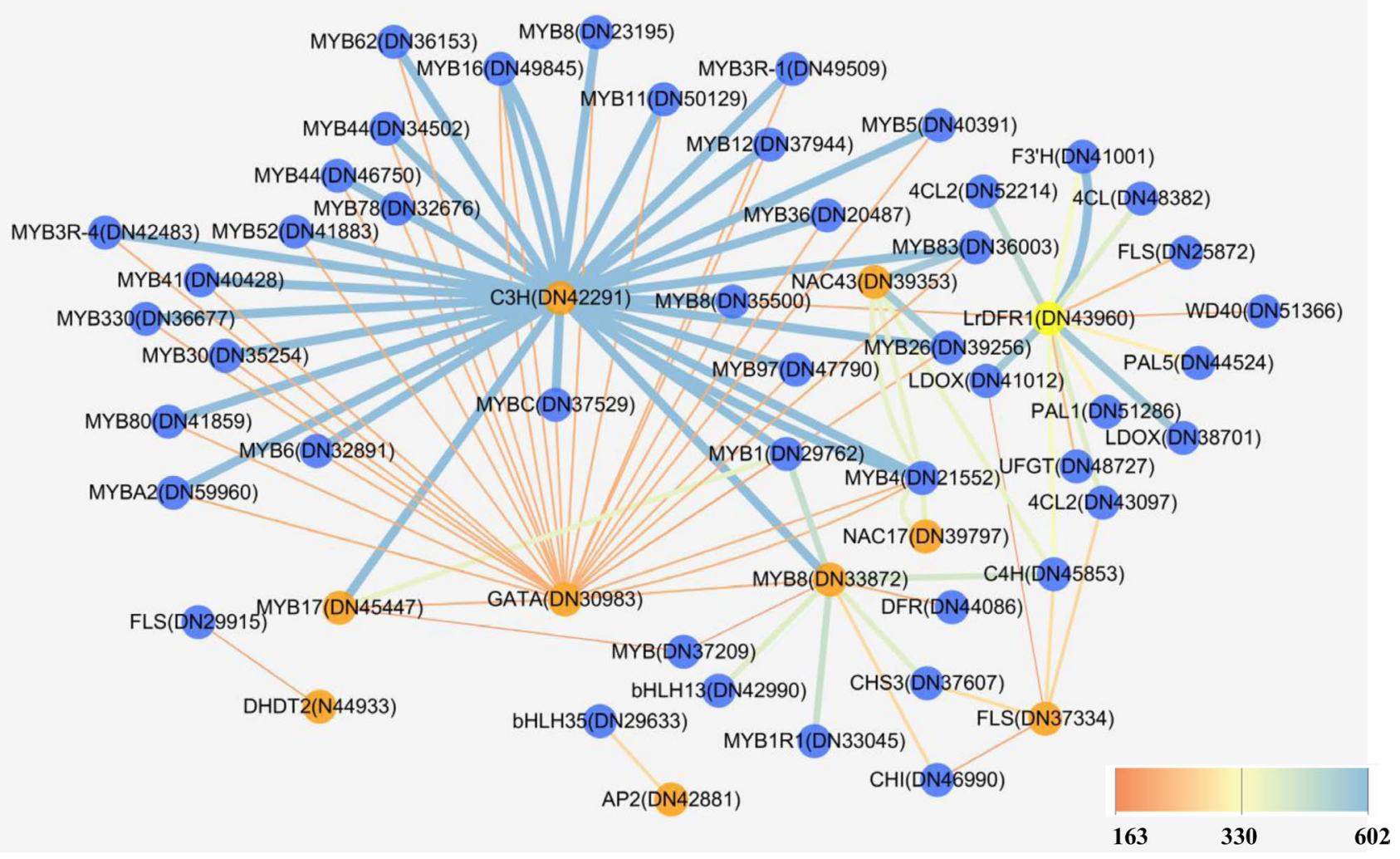

FIGURE 5 | Gene expression profiles of identified transcription factor and protein-protein interaction network of key structural enzymes and TFs involved in anthocyanin biosynthesis in $L$. radiata flowers. (A) $K$-means clusters of DEGs based on standardized ( $\log _{2}$ transformed) FPKM of $L$. radiata petals at flower development stage (FB, FL1, FL2, and R). Number of genes that were clustered in every subclass are shown above each Figure. (B) Protein-protein interaction network constituted by protein sequences of differentially expressed transcription factors and structural genes involved in anthocyanin synthesis of $L$. radiata petals. Genes that have the higher weight are depicted in 'yellow and orange,' the 'blue edges' correspond to co-expressed strong links and the 'yellow edges' correspond to co-expressed weak links. 

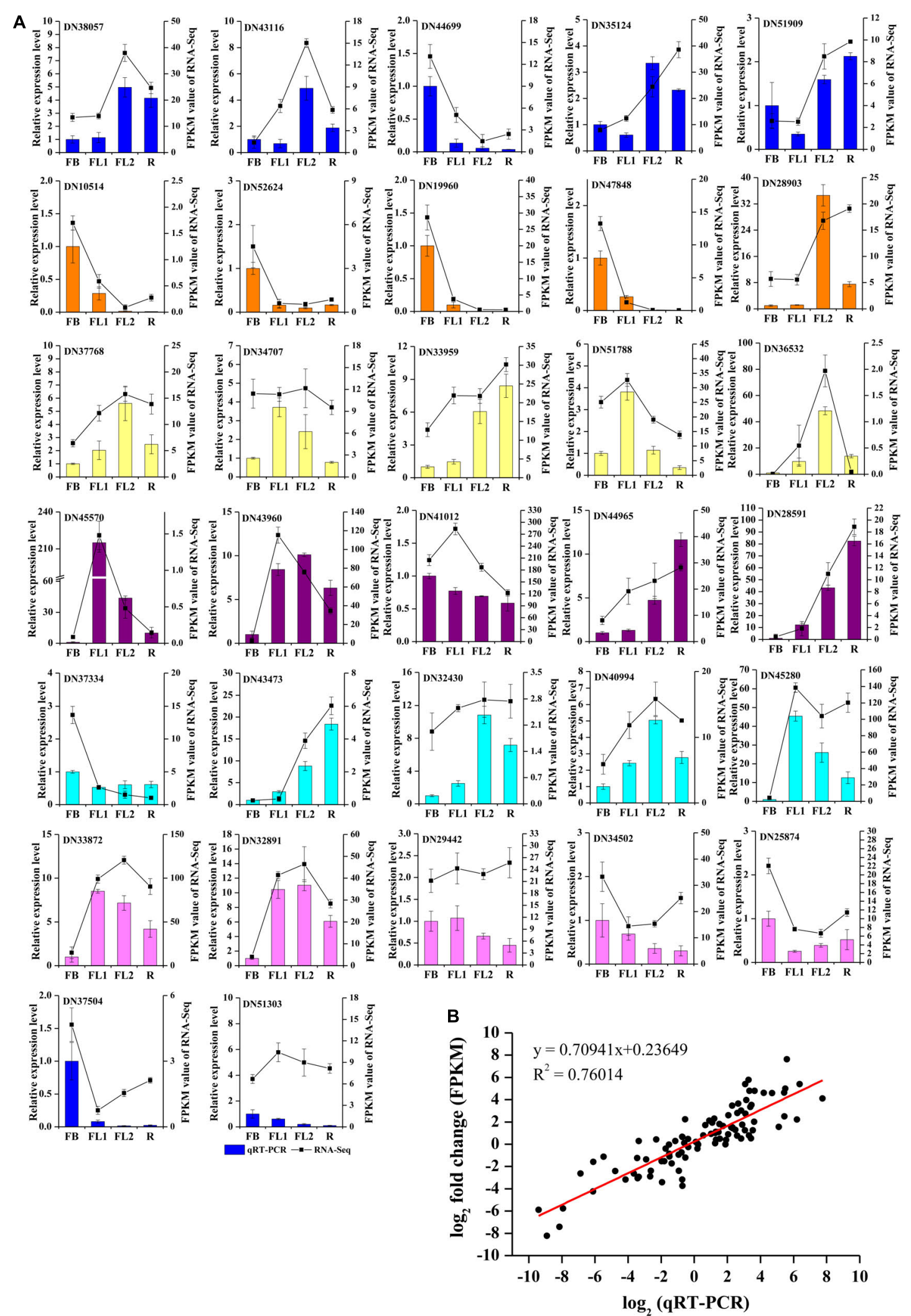

FIGURE 6 | qRT-PCR validation of gene expression level in the transcriptome. (A) qRT-PCR validation of gene expression level in the transcriptome. Thirty two unigenes were selected for qRT-PCR validation. (B) Correlation analysis of the results between qRT-PCR and RNA-Seq. 


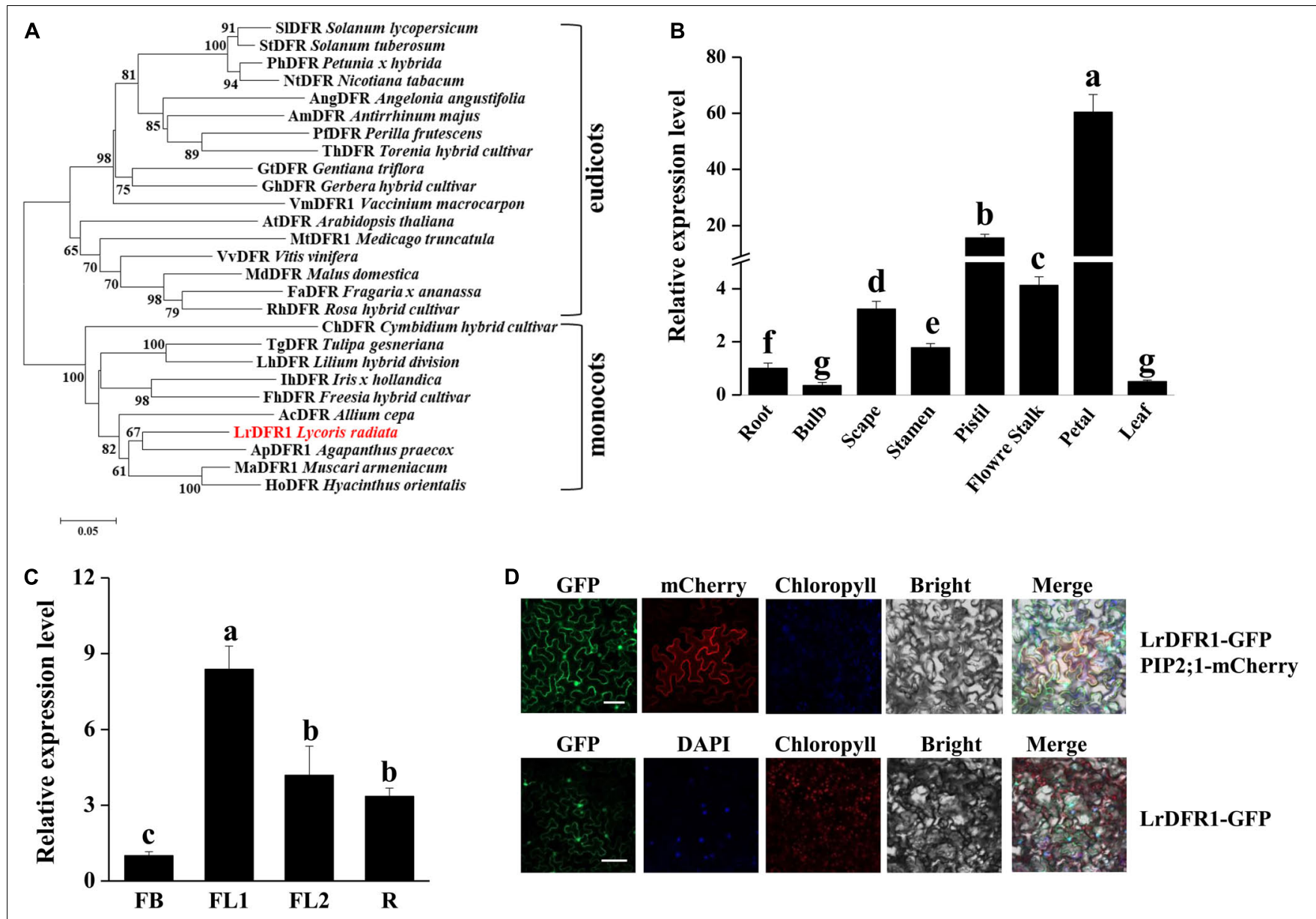

FIGURE 7 | Phylogenetic tree analysis, transcription expression profiles, and subcellular localization of LrDFR1. (A) Phylogenetic assessment of LrDFR1 with other DFR proteins from different plants. Phylogenetic tree generation was achieved using the maximum likelihood method in MEGA 7.0 software. Numbers at every interior branch show bootstrap values of 1000 replicates. The bar shows a 0.05 genetic distance. Plant species as well as GenBank accession numbers of DFR proteins used in phylogenetic analyses are: Solanum tuberosum StDFR (AF449422), Solanum lycopersicum SIDFR (CAA79154.1), Petunia hybrida PhDFR (AF233639), Angelonia angustifolia AngDFR (KJ817183), Nicotiana tabacum NtDFR (NP_001312559.1), Antirrhinum majus AmDFR (X15536), Perilla frutescens PfDFR (AB002817), Gentiana triflora GtDFR (D85185), Torenia hybrid ThDFR (AB012924), Gerbera hybrid GhDFR (Z17221), Vaccinium macrocarpon VmDFR1 (AF483835), Arabidopsis thaliana AtDFR (AB033294), Medicago truncatula MtDFR1 (AY389346), Vitis vinifera VvDFR (Y11749), Malus domestica MdDFR (AAO39816), Rosa hybrid RhDFR (D85102), Cymbidium hybrid ChDFR (AF017451), Fragaria ananassa FaDFR (AF029685), Tulipa gesneriana TgDFR (BAH98155.1), Lilium hybrid LhDFR (AB058641), Iris hollandica IhDFR (BAF93856.1), Allium cepa AcDFR (AY221250.2), Agapanthus praecox ApDFR (AB099529.1), Muscari aucheri MaDFR (MH636605), Freesia hybrid FhDFR (KU132389), and Hyacinthus orientalis HoDFR (AFP58815.1). (B) Expression profiles of LrDFR1 in various tissues of L. radiata. Expressions of LrDFR1 were assessed by qRT-PCR, and normalized to LrTIP41. Expressions of LrDFR1 in root tissues were defined as 1.0. Data are shown as mean \pm SD. Bars with different letters are significantly different at $p<0.05$ according to Duncan's multiple range test. (C) Expression profiles of $L r D F R 1$ during the FB stage, FL1 stage, FL2 stage and R stage of $L$. radiata. Expression levels of LrDFR1 were assessed by qRT-PCR, and normalized to $L r T I P 41$. Expression levels of LrDFR1 in FB stage were defined as 1.0. Data are shown as mean \pm SD. Bars with different letters are significantly different at $p<0.05$ according to Duncan's multiple range test. (D) Subcellular localization of LrDFR1 in N. benthamiana epidermal cells. Scale bars $=20 \mu \mathrm{m}$. The nuclei are indicated by DAPI staining.

The deduced amino acid sequence of LrDFR1 revealed a high similarity with DFR proteins from Agapanthus praecox (75.33\%), Muscari armeniacum (74.74\%), and Hyacinthus orientalis (72.72\%) (Figure 7A). Multiple amino acid sequence alignments showed the highly preserved NADPH-binding motif (VTGAAGFIGSWLIMRLLERGY) (Gang, 2005) and the substrate-binding domain (T128-K154) (Johnson et al., 2001) in the LrDFR1 sequence (Supplementary Figure 11). qRTPCR was then performed to assess whether expression patterns of LrDFR1 in different tissues and flower development stages were coincided with anthocyanin accumulation in $L$. radiata.
LrDFR1 was found to be expressed in all tissues, with the highest expression levels in petals (Figure 7B). Moreover, expression levels of LrDFR1 were significantly increased from stage FB to stage R, peaking at stage FL1 (Figure 7C). These findings imply tissue-specific expression levels for $\operatorname{LrDFR} 1$, which is associated with anthocyanin accumulation in $L$. radiata petals.

Moreover, we transiently expressed LrDFR1 in tobacco epidermal cells to assess subcellular localization of LrDFR1. As shown in Figure 7D, the fluorescent signal of LrDFR1-GFP was localized into the nucleus, cytoplasm and cell membrane, while GFP was evenly distributed in the cell (Figure 7D 


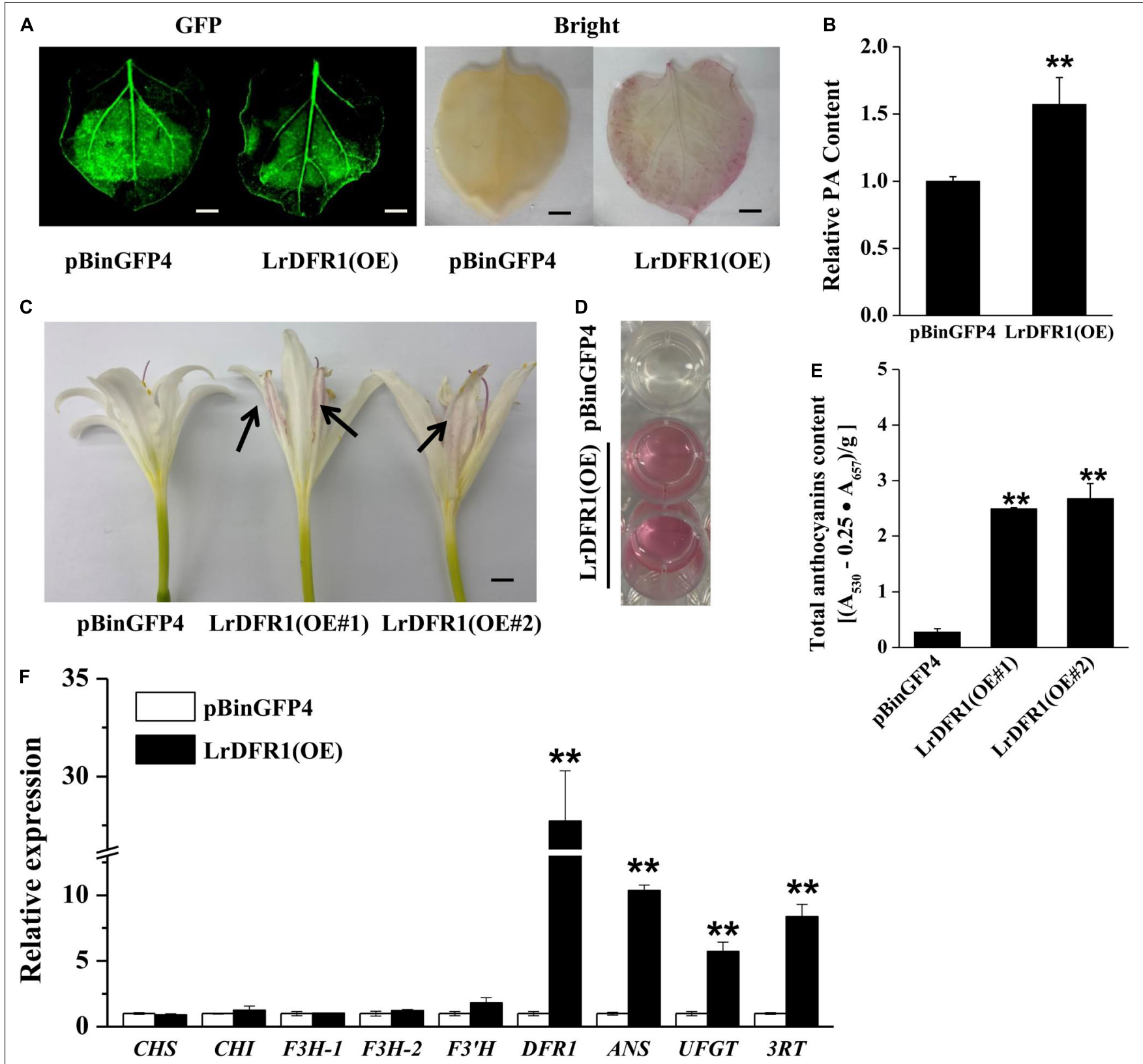

FIGURE 8 | Overexpression of LrDFR1 promotes anthocyanin and proanthocyanidin biosynthesis. (A) Proanthocyanidin staining and (B) relative proanthocyanidin (PA) levels in transiently transformed tobacco leaves (pBinGFP4: empty vector controls; LrDFR1-OE: LrDFR1-overexpressing leaves). Tobacco leaves were kept in a phytotron at $24^{\circ} \mathrm{C}$ under constant lighting for 5 days. DMACA was used to stain proanthocyanidin. Every experiment was performed using $8-10$ leaves for each genotype. Experiments were conducted in triplicates, and a representative image is shown. Proanthocyanidin levels of empty vector controls were set as the reference to 1. Asterisks represent significant differences between control and LrDFR1-overexpressing leaves $\left({ }^{\star \star} p<0.01\right)$. Bars $=1 \mathrm{~cm}$. (C) Phenotypes of anthocyanin accumulation. Arrow indicates the transfected petals. (D,E) Relative anthocyanin levels in transiently transformed Lycoris petals (pBinGFP4: empty vector controls; LrDFR1-OE: LrDFR1-overexpressing petals). Lycoris petals were kept in a phytotron at $24^{\circ} \mathrm{C}$ with a constant light for 5 days. Every experiment was performed using 8-10 petals per genotype. Data are shown as mean $\pm \mathrm{SD}$. ${ }^{\star \star} p<0.01$. Bar $=0.5 \mathrm{~cm}$. (F) Relative expression levels of endogenous anthocyanin biosynthetic genes in pBinGFP4 (empty vector controls) as well as $L r D F R 1$-overexpressing petals. Expression patterns of early biosynthetic genes (CHS, F3H, $\mathrm{CHI}$, and $F 3^{\prime} H$ ) as well as late biosynthetic genes (DFR, UFGT, ANS, and 3RT) in petals were investigated. Asterisks represent significant differences between control and LrDFR1-overexpressing petals $\left({ }^{\star \star} p<0.01\right)$.

and Supplementary Figure 12). To determine the roles of LrDFR1 in regulating anthocyanin as well as proanthocyanidin biosynthesis in L. radiata, an LrDFR1-overexpressing plasmid was transfected into Lycoris petals and tobacco epidermal cells
(Figure 8A). Overexpression of LrDFR1 in tobacco and Lycoris petals markedly enhanced proanthocyanidin and anthocyanin accumulation (Figures 8B-E). To assess the effects of LrDFR 1 on endogenous Lycoris petals genes that are involved in anthocyanin 
synthesis, the expression levels of $C H S, C H I, F 3 H, F 3^{\prime} H, D F R$, ANS, UFGT, and 3RT were determined (Figure 8F). Among them, the expressions of LrDFR1, ANS, UFGT and $3 R T$ were significantly higher in $L r D F R 1$-overexpressing plants than in control plants (Figure 8F). These results suggest that LrDFR1 may play important roles in anthocyanins biosynthesis of Lycoris petals.

\section{DISCUSSION}

\section{Changes of Anthocyanin Contents in the Lycoris radiata Petals During Flower Development Stages}

Flowering plants exhibit a wide variation in their flora, foliage, and fruit colors, as a result of genetic factors and variations in environments. Flavonoids/anthocyanins, betalains and carotenoids are the major metabolites for coloration in plant reproductive organs (Griesbach, 2005; Tanaka et al., 2008). Most of the red, purple, and blue-colored flowers (such as red rose, lavender, and blue chicory) as well as fruits (such as berries, currants, and grapes) contained high anthocyanins content (Khoo et al., 2017). The genus Lycoris is used as a garden flower due to the colorful and special flowers, and the flower colors of Lycoris are diverse. For example, the flower color of L. radiata and $L$. rosea was red, that of L. aurea and L. chinensis was yellow. L. sprengeri and L. haywardii showed red and blue color, while L. longituba displays an exceptionally wide diversity of flower colors from purple, red, orange, to yellow (He et al., 2011). Similar to the flowers of other species, the petals of Lycoris are rich in anthocyanins, and their color formation are largely related to anthocyanins (He et al., 2011; Chun et al., 2013; Yue et al., 2019; Park et al., 2021). In this study, we determined the content of anthocyanins in the petals of $L$. radiata, and the results showed that the color intensity of the L. radiata petals was changed with the different anthocyanin contents. The anthocyanins increased then decreased during the flower development stages (Figure 1), which are similar to the results recently reported by Park et al. (2021).

\section{Key Structural Genes Responsible for Anthocyanin Synthesis in Lycoris radiata Petals During Flower Development Stages}

To data, transcriptome sequencing is highly employed for predicting novel genes, gene function, and genome evolution for plant breeding and horticulture research (Rameneni et al., 2020). For example, transcriptome analysis has revealed the role of anthocyanin in flower color formation in several horticultural crops, such as Camellia sinensis (Zhou et al., 2020), "Tiny Padhye" (Lilium spp.) (Xu et al., 2017), lilies (Lilium spp.) (Suzuki et al., 2016), Magnolia sprengeri (Shi et al., 2014), Paeonia lactiflora (Zhao et al., 2014), Paeonia delavayi (Shi et al., 2015), and Silene littorea (Casimiro-Soriguer et al., 2016). For better understanding of petals color formation during flower development stages in L. radiata, a comparative transcriptomics analysis was carried out. The results showed that approximately 70.27 GB of highquality data, and 87,584 unigenes were obtained. Further analyses, based on NR, Swiss-Prot, KEGG, KOG, GO, Pfam, and eggNOG databases, predicted 38,798 DEGs associated with a specific or general function (Supplementary Tables 4, 5).

The variations in floral coloration emanates from different processes, such as pathways competition, expression levels of structural genes involved in pigment formation, and mutations of structural or regulatory genes (Grotewold, 2006; Cui et al., 2021). In plants, phenylpropanoids represent a vital group of physiologically active secondary metabolites derived from phenylalanine, and anthocyanins, flavonols, isoflaconoids and flavonols have a similar metabolism pathway during their biosynthesis (Ferrer et al., 2008). KEGG pathway analysis showed that the 'phenylpropanoid biosynthesis,' 'flavonoid biosynthesis,' as well as 'flavone and flavonol biosynthesis' pathways were enriched between each two transcriptomes of $L$. radiata petals during flower development stages (Figure $3 \mathbf{B}$ and Supplementary Figure 8). Given that anthocyanin biosynthesis pathway is well known to modulate color formation in plants, we mainly focused on them as the candidate pathways to elucidate their involvement in petal/flower color formation in L. radiata. Subsequently, we identified the main functional genes participated in the anthocyanin biosynthetic pathway, and found that most of structural genes such as $F 3^{\prime} H, U F G T$, $D F R$, and FLS were elevated in L. radiata petals at FL1 and FL2 stages (Figure 4A). Therefore, these genes might have contributed to the increasing anthocyanin content in petals from the FB stage to the FL1 and FL2 stage, as evidenced in Figure 1. For example, three $F 3^{\prime} H$ genes (DN41001, DN43758, and DN46768) were highly expressed in petals at FL1 and FL2 stages (Figure 4A). Another prominent gene, UFGT (DN44965), which glycolyzes anthocyanidin into anthocyanin (Xie et al., 2003), was also highly expressed in petals at FL1, FL2 and $\mathrm{R}$ stages, as compared to that of the samples at $\mathrm{FB}$ stage (Figure 4A). All of these genes were positively correlated with the biosynthesis of anthocyanins (Niu et al., 2010). Notably, two DFR genes (DN42380, DN43960) and one FLS (DN37334) (Table 1) were found to be highly associated with the total anthocyanins content $(|\mathrm{PCC}|>0.8)$, suggesting they may have an essential function in the phenotypic expression of petal color (Figure 4B). In anthocyanin biosynthesis, DFR catalyze the reduction of dihydroquercetin to leucoanthocyanidins, and the level of DFR expression have been associated with flower color changes (Nakatsuka et al., 2003; Zhao et al., 2012). qRT-PCR also indicated that the hub gene $L r D F R 1$ were mostly expressed the most in the FL1 samples (Figure 7C). Our results suggest that these enzymes may be the most important enzymes to catalyze anthocyanin biosynthesis in L. radiata petals.

\section{Transcriptional Regulation of Color Formation in Lycoris radiata Petals}

Transcription factors play critical functions in flavonoid biosynthesis, by regulating expression of structural genes. For example, the class of TFs identified were previously implicated in regulation of petal color formation in roses 
(Li D. et al., 2020). Particularly, MYB-bHLH-WD40 complexes have been implicated in multi-level regulation of flavonoid biosynthesis (Gallego et al., 2018), whereas the R2R3-MYB family was shown to play a vital role in regulation of spatiotemporal expressions of genes involved in anthocyanin biosynthetic in plants (Gonzalez et al., 2008; Zhao and Tao, 2015). Besides, MYB-domain TFs are important mediators of anthocyanin accumulation and participate in colorations of various organs in horticultural as well as ornamental plants (Tang et al., 2017; Liu Y. et al., 2019; Xiang et al., 2019; Zhai et al., 2019; He et al., 2020; Sun et al., 2020; Wang et al., 2020; Zhong et al., 2020).

In this study, the most abundant TFs including $A P 2 / E R F$, bHLH, bZIP, C2C2, HSF, MYB, NAC, TIFY, and WRKY families were predicted (Supplementary Table 8). In addition, we employed a $K$-means clustering, as proposed earlier by Handhayani and Hiryanto (2015), which permitted the clustering of 272 TF unique genes among the samples (FB, FL1, FL2, and R) into six sub-clusters with some members in Cluster 2 associated with genes from the MYB and $b H L H$ TFs (Figure 5A). Based on the expression level of TFs obtained from the transcriptome data, 27 TFs (Table 1) were found to highly associate with the total anthocyanin content (|PCC| $>0.8$ ), and these TFs may have an essential function in the phenotypic expression of L. radiata petal color. Interestingly, among these TFs, three MYBs showed two different expression patterns. The expression level of two MYBs (DN45447, DN51496) was highest in FB, followed by FL1, FL2, and R, which was contrary to the total anthocyanin content trend. Conversely, the expression of LrMYB1 (DN33872) exhibited a similar trend to the total anthocyanin content in the $L$. radiata petals (Supplementary Figure 13), indicating that MYBs (DN45447 and DN51496) negatively regulated anthocyanin accumulation, whereas LrMYB1 (DN33872) was identified as one of the eight hub genes may positively regulate anthocyanin accumulation in L. radiata (Table 1 and Figure 5B).

Subsequently, two negatively correlated $b H L H s$ (DN36174 and DN41224) and one positively correlated LrbHLH1 (DN48856) were identified (Table 1). In plants, MYB often forms protein complexes with bHLH and WD40 to participate in anthocyanin biosynthesis rather than regulate anthocyanin biosynthesis directly (Feng et al., 2020). In apple, MdMYB1, MdMYB9, $M d M Y B 10$, and $M d M Y B A$ act as positive modulators of anthocyanin biosynthesis, by activating the expressions of MdDFR and MdUF3GT (Takos et al., 2006; Ban et al., 2007; Espley et al., 2007; An et al., 2015). On the contrary, downregulation of $M d M Y B 1$ inhibits anthocyanin accumulation mediated by ethylene, abscisic acid (ABA), wounding, drought, and different light intensities (An et al., 2018, 2019, 2020a,b). Notably, our results also revealed a significant upregulation of LrMYB1 (DN33872) and LrbHLH1 (DN48856), of which the expression was positively correlated with $\operatorname{LrDFR} 1, \mathrm{LrCHS}, \mathrm{LrCHI}$, $F 3^{\prime} H$, LrUFGT and LrANS genes during petal development stages (Supplementary Figure 13). This is similar to that of LhMYB12Lat, which has previously been associated with activation of accumulation of anthocyanin in lily petals (Yamagishi et al., 2014). In our co-expression networks, the module that was positively correlated with anthocyanin contents and modules negatively correlated with anthocyanin content were identified. Overall, whether these MYB TFs interact with $b H L H$ TFs to regulate anthocyanin biosynthesis in $L$. radiata remains to be further investigated.

\section{The LrDFR1 Drives Anthocyanin Accumulation in Lycoris radiata Petals}

In the anthocyanin biosynthesis pathway, DFR catalyzes dihydroflavonol conversion to leucoanthocyanidins (Zhang et al., 2014). DFR belongs to the superfamily of short chain dehydrogenase reductase (SDR), which has a highly preserved NADPH-binding domain "VTGAAGFIGSWLIMRLLERGY" as well as a substrate-binding domain in plants (Martens et al., 2002; Haselmair-Gosch et al., 2018). In this study, based on the expression level of the anthocyanin structure genes obtained from the transcriptome data, LrDFR1 and LrDFR2 (Table 1) were found to highly associate with the total anthocyanin content (PCC $>0.8)$, suggesting DFR may have an essential function in the phenotypic expression of $L$. radiata petal color. LrDFR1 was then identified as one of the hub genes (Figure 5B) and important to positively regulate anthocyanin production in L. radiata petals. Multiple amino acid alignments showed that LrDFR1 contains the NADPH-binding domains and substrate-binding domains. Phylogenic tree analysis revealed a high similarity between LrDFR1 and other characterized DFRs, implying that LrDFR1 belongs to the monocot DFR family and exhibits catalytic characteristics.

The DFR genes of Iris and Gentiana have been reported to be associated with the absence of brick-red flowers (Noda et al., 2017). Moreover, heterologous MaDFR expressions in $N$. tabacum has been associated with enhanced anthocyanin accumulation, which leads to darker flower colors, suggesting that $M a D F R$ is involved in flower color development (Liu H. et al., 2019). After the introduction of maize (Zea mays) $D F R$ into white-flowered petunia varieties, transgenic plant flowers accumulate non-native pelargonidin, which results in novel brick red-flower varieties (Meyer et al., 1987). In this study, the expression patterns of LrDFR1 was first temporally and spatially tested in various tissues and petal development stages of $L$. radiata. It showed that the expression levels of LrDFR1 were correlated with total anthocyanin accumulation. These findings imply that LrDFR1 is associated with petal color development in L. radiata (Figures 1, 7C,D). The spatial and temporal expression characteristics of LrDFR1 gene were found similarly in several other species (Liu H. et al., 2019; Lim et al., 2020). In order to investigate the functional divergence of LrDFR1 gene in the flavonoid biosynthesis, we performed transient expression analyses using Lycoris petals and tobacco leaves. Overexpressed LrDFR1 was associated with significantly elevated anthocyanin content and proanthocyanidin content in Lycoris petals and tobacco leaves. Interestingly, overexpression of LrDFR1 also enhanced the expression of downstream genes (LrANS and LrUFGT) involved in anthocyanins biosynthesis in transgenic Lycoris petals (Figures 8B-F). In addition, for plant breeders, a single DFR gene maybe ideal for determining flower colors. DFR is vital for pigmentation, when compared to other 
anthocyanin biosynthetic genes, which only regulate plant flower color hue. Thus, whether LrDFR1 has a high preference for dihydromyricetin, and is accountable for the limited flower colors in $L$. radiata needs further study.

\section{CONCLUSION}

In this study, we provided a dynamic transcriptome profile of $L$. radiata petals during flower development stages. Overall, 56 structural genes and 27 key TF DEGs were identified as key genes responsible for $L$. radiata petal coloration. In the protein-protein interaction network analysis, LrDFR1 was identified as a hub gene in the anthocyanin biosynthesis pathway, and was highly associated with anthocyanin accumulation. Overexpression of LrDFR1 in Lycoris petals and tobacco leaves induced anthocyanin accumulation. In addition, the structural genes and co-expressed TFs reported in this study would serve as useful genetic resources for further functional characterization and molecular breeding programs in L. radiata. Taken together, our results elucidate on the molecular basis of petal development in $L$. radiata.

\section{DATA AVAILABILITY STATEMENT}

The datasets presented in this study can be found in online repositories. The names of the repository/repositories and accession number(s) can be found below: https://ngdc.cncb.ac. $\mathrm{cn} /$, CRA004779.

\section{REFERENCES}

An, J. P., Liu, Y. J., Zhang, X. W., Bi, S. Q., Wang, X. F., You, C. X., et al. (2020a). Dynamic regulation of anthocyanin biosynthesis at different light intensities by the BT2-TCP46-MYB1 module in apple. J. Exp. Bot. 71, 3094-3109. doi: 10.1093/jxb/eraa056

An, J. P., Zhang, X. W., Bi, S. Q., You, C. X., Wang, X. F., and Hao, Y. J. (2020b). The ERF transcription factor MdERF38 promotes drought stressinduced anthocyanin biosynthesis in apple. Plant J. 101, 573-589. doi: 10.1111/ tpj. 14555

An, J. P., Qu, F. J., Yao, J. F., Wang, X. N., You, C. X., Wang, X. F., et al. (2017). The bZIP transcription factor MdHY5 regulates anthocyanin accumulation and nitrate assimilation in apple. Hortic. Res. 4:17023. doi: 10.1038/hortres.2017.56

An, J. P., Wang, X. F., Li, Y. Y., Song, L. Q., Zhao, L. L., You, C. X., et al. (2018). EIN3-LIKE1, MYB1, and ethylene response factor 3 act in a regulatory loop that synergistically modulates ethylene biosynthesis and anthocyanin accumulation. Plant Physiol. 178, 808-823.

An, J. P., Wang, X. F., Zhang, X. W., Bi, S. Q., You, C. X., and Hao, Y. J. (2019). MdBBX22 regulates UV-B-induced anthocyanin biosynthesis through regulating the function of MdHY5 and is targeted by MdBT2 for 26S proteasome-mediated degradation. Plant Biotechnol. J. 17, 2231-2233. doi: 10. 1104/pp.18.00068

An, X. H., Tian, Y., Chen, K. Q., Liu, X. J., Liu, D. D., Xie, X. B., et al. (2015). MdMYB9 and MdMYB11 are involved in the regulation of the JA-induced biosynthesis of anthocyanin and proanthocyanidin in apples. Plant Cell Physiol. 56, 650-662. doi: 10.1093/pcp/pcu205

Ban, Y., Honda, C., Hatsuyama, Y., Igarashi, M., Bessho, H., and Moriguchi, T. (2007). Isolation and functional analysis of a MYB transcription factor gene that is a key regulator for the development of red coloration in apple skin. Plant Cell Physiol. 48, 958-970. doi: 10.1093/pcp/ pcm066

\section{AUTHOR CONTRIBUTIONS}

NW and ZW designed the research and wrote the manuscript. NW performed most of the experiments and data analysis. XS and FZ collected the experimental materials. TW assisted with the data analysis. WZ provided helpful comments on the manuscript. ZW provided guidance on the whole study and contributed with valuable discussions. All authors read and approved the final manuscript.

\section{FUNDING}

This research was funded by the Jiangsu Key Laboratory for the Research and Utilization of Plant Resources (Grant No. JSPKLB202020). This work was jointly funded by Projects of Independently Development of Jiangsu Provincial Department of Science and Technology (BM2018021-5); Jiangsu Agricultural Science and Technology Innovation Fund [Grant Nos. CX(20)3171 and CX(19)3033]; the National Natural Science Foundation of China under Grant (Grant No. 31801900); and the Natural Science Foundation of Jiangsu Province (Grant No. BK20180310).

\section{SUPPLEMENTARY MATERIAL}

The Supplementary Material for this article can be found online at: https://www.frontiersin.org/articles/10.3389/fpls.2021. 761862/full\#supplementary-material

Baudry, A., Caboche, M., and Lepiniec, L. (2006). TT8 controls its own expression in a feedback regulation involving TTG1 and homologous MYB and bHLH factors, allowing a strong and cell-specific accumulation of flavonoids in Arabidopsis thaliana. Plant J. 46, 768-779. doi: 10.1111/j.1365-313X.2006. 02733.x

Casimiro-Soriguer, I., Narbona, E., Buide, M. L., Del Valle, J. C., and Whittall, J. B. (2016). Transcriptome and biochemical analysis of a flower color polymorphism in Silene littorea (Caryophyllaceae). Front. Plant Sci. 7:204. doi: 10.3389/fpls.2016.00204

Castaneda-Ovañdo, A., Pacheco-Hernández, M. D., Páez-Hernández, M. E., Rodríguez, J. A., and Galán-Vidal, C. A. (2009). Chemical studies of anthocyanins: a review. Food Chem. 113, 859-871. doi: 10.1016/j.foodchem. 2008.09.001

Chun, J. H., Jang, I. H., Arasu, M. V., Aldhabi, N. A., Duraipandiyan, V., Lee, D. H., et al. (2013). Isolation and identification of alkaloids and anthocyanins from flower and bulb of Lycoris radiata using HPLC and LC-ESI-MS. J. Agric. Chem. Environ. 1, 22-2610.

Cui, X., Deng, J., Huang, C., Tang, X., Li, X., Li, X., et al. (2021). Transcriptomic analysis of the anthocyanin biosynthetic pathway reveals the molecular mechanism associated with purple color formation in Dendrobium Nestor. Life 11:113. doi: 10.3390/life11020113

Dong, Q., Zhao, H., Huang, Y., Chen, Y., Wan, M., Zeng, Z., et al. (2020). FtMYB18 acts as a negative regulator of anthocyanin/proanthocyanidin biosynthesis in Tartary buckwheat. Plant Mol. Biol. 104, 309-325. doi: 10.1007/s11103-02001044-5

Espley, R. V., Hellens, R. P., Putterill, J., Stevenson, D. E., Kutty-Amma, S., and Allan, A. C. (2007). Red colouration in apple fruit is due to the activity of the MYB transcription factor, MdMYB10. Plant J. 49, 414-427. doi: 10.1111/j.1365313X.2006.02964.x

Fang, H. C., Dong, Y. H., Yue, X. X., Hu, J. F., Jiang, S. H., Xu, H. F., et al. (2019). The B-box zinc finger protein MdBBX20 integrates anthocyanin accumulation 
in response to ultraviolet radiation and low temperature. Plant Cell Environ. 42, 2090-2104. doi: 10.1111/pce.13552

Feng, C., Ding, D., Feng, C., and Kang, M. (2020). The identification of an R2R3MYB transcription factor involved in regulating anthocyanin biosynthesis in Primulina swinglei flowers. Gene 752:144788. doi: 10.1016/j.gene.2020.144788

Feng, X., Zhang, Y., Wang, H., Tian, Z., Xin, S., and Zhu, P. (2021). The dihydroflavonol 4-reductase BoDFR1 drives anthocyanin accumulation in pink-leaved ornamental kale. Theor. Appl. Genet. 134, 159-169. doi: 10.1007/ s00122-020-03688-9

Ferrer, J.-L., Austin, M. B., Stewart, C. Jr., and Noel, J. P. (2008). Structure and function of enzymes involved in the biosynthesis of phenylpropanoids. Plant Physiol. Biochem. 46, 356-370. doi: 10.1016/j.plaphy.2007.12.009

Francavilla, A., and Joye, I. J. (2020). Anthocyanins in whole grain cereals and their potential effect on health. Nutrients 12:2922. doi: 10.3390/nu12102922

Gallego, A. M., Rojas, L. F., Parra, O., Rodriguez, H. A., Mazo Rivas, J. C., Urrea, A. I., et al. (2018). Transcriptomic analyses of cacao cell suspensions in light and dark provide target genes for controlled flavonoid production. Sci. Rep. 8:13575. doi: 10.1038/s41598-018-31965-7

Gang, D. R. (2005). Evolution of flavors and scents. Annu. Rev. Plant Biol. 56, 301-325. doi: 10.1146/annurev.arplant.56.032604.144128

Gerats, T., and Strommer, J. (eds) (2009). "Petunia: evolutionary, developmental and physiological genetics," in Petunia, (New York, NY: Springer). doi: 10.1007/ 978-0-387-84796-2

Gonzalez, A., Zhao, M., Leavitt, J. M., and Lloyd, A. M. (2008). Regulation of the anthocyanin biosynthetic pathway by the TTG1/bHLH/Myb transcriptional complex in Arabidopsis seedlings. Plant J. 53, 814-827. doi: 10.1111/j.1365313X.2007.03373.x

Grabherr, M. G., Haas, B. J., Yassour, M., Levin, J. Z., Thompson, D. A., Amit, I., et al. (2011). Full-length transcriptome assembly from RNA-Seq data without a reference genome. Nat. Biotechnol. 29, 644-652. doi: 10.1038/nbt.1883

Griesbach, R. J. (2005). "Biochemistry and genetics of flower color," in Plant Breeding Reviews, ed. J. Janick (Hoboken, NJ: Wiley-Blackwell), 89-114. doi: 10.1002/9780470650301.ch4

Grotewold, E. (2006). The genetics and biochemistry of floral pigments. Annu. Rev. Plant Biol. 57, 761-780. doi: 10.1146/annurev.arplant.57.032905.105248

Handhayani, T., and Hiryanto, L. (2015). Intelligent Kernel K-means for clustering gene expression. Procedia Comput. Sci. 59, 171-177. doi: 10.1016/j.procs.2015. 07.544

Haselmair-Gosch, C., Miosic, S., Nitarska, D., Roth, B. L., Walliser, B., Paltram, R., et al. (2018). Great cause-small effect: undeclared genetically engineered orange petunias harbor an inefficient dihydroflavonol 4-reductase. Front. Plant Sci. 9:149. doi: 10.3389/fpls.2018.00149

He, C., Liu, X., Teixeira da Silva, J. A., Liu, N., Zhang, M., and Duan, J. (2020). Transcriptome sequencing and metabolite profiling analyses provide comprehensive insight into molecular mechanisms of flower development in Dendrobium officinale (Orchidaceae). Plant Mol. Biol. 104, 529-548. doi: 10. 1007/s11103-020-01058-z

He, Q. L., Cui, S. J., Gu, J. L., Zhang, H., Wang, M. X., Zhou, Y., et al. (2011). Analysis of floral transcription factors from Lycoris longituba. Genomics 96, 119-127. doi: 10.1016/j.ygeno.2010.04.002

Hsu, C. C., Chen, Y. Y., Tsai, W. C., Chen, W. H., and Chen, H. H. (2015). Three R2R3-MYB transcription factors regulate distinct floral pigmentation patterning in Phalaenopsis spp. Plant Physiol. 168, 175-191. doi: 10.1104/pp. 114.254599

Huang, Y., Xuan, H., Yang, C., Guo, N., Wang, H., Zhao, J., et al. (2019). GmHsp90A2 is involved in soybean heat stress as a positive regulator. Plant Sci. 295, 26-33. doi: 10.1016/j.plantsci.2019.04.016

Jiang, G., Li, Z., Song, Y., Zhu, H., Lin, S., Huang, R., et al. (2019). LcNAC13 Physically interacts with LcR1MYB1 to coregulate anthocyanin biosynthesisrelated genes during Litchi fruit ripening. Biomolecules 9:135. doi: 10.3390/ biom 9040135

Jiang, L., Fan, Z., Tong, R., Zhou, X., Li, J., and Yin, H. (2020). Functional diversification of the dihydroflavonol 4-reductase from Camellia nitidissima Chi. in the control of polyphenol biosynthesis. Genes 11:1341. doi: 10.3390/ genes11111341

Jin, S. W., Rahim, M. A., Afrin, K. S., Park, J. I., Kang, J. G., and Nou, I. S. (2018). Transcriptome profiling of two contrasting ornamental cabbage (Brassica oleracea var. acephala) lines provides insights into purple and white inner leaf pigmentation. BMC Genomics 19:797. doi: 10.1186/s12864-018-5199-3

Jin, S. W., Rahim, M. A., Jung, H. J., Afrin, K. S., Kim, H. T., Park, J. I., et al. (2019). Abscisic acid and ethylene biosynthesis-related genes are associated with anthocyanin accumulation in purple ornamental cabbage (Brassica oleracea var. acephala). Genome 62, 513-526. doi: 10.1139/gen-2019-0038

Johnson, E. T., Ryu, S., Yi, H., Shin, B., Cheong, H., and Choi, G. (2001). Alteration of a single amino acid changes the substrate specificity of dihydroflavonol 4-reductase. Plant J. 25, 325-333. doi: 10.1046/j.1365-313x.2001.00962.x

Khoo, H. E., Azlan, A., Tang, S. T., and Lim, S. M. (2017). Anthocyanidins and anthocyanins: colored pigments as food, pharmaceutical ingredients, and the potential health benefits. Food Nutr. Res. 61:1361779. doi: 10.1080/16546628. 2017.1361779

Koes, R., Verweij, W., and Quattrocchio, F. (2005). Flavonoids: a colorful model for the regulation and evolution of biochemical pathways. Trends Plant Sci. 10, 236-242. doi: 10.1016/j.tplants.2005.03.002

Lev-Yadun, S., and Gould, K. S. (2009). "Role of Anthocyanins in Plant Defence," in Anthocyanins, ed. C. Winefield, K. Davies and K. Gould K (Cham: Springer), 22-28.

Li, C., Qiu, J., Ding, L., Huang, M., Huang, S., Yang, G., et al. (2017). Anthocyanin biosynthesis regulation of DhMYB2 and DhbHLH1 in Dendrobium hybrids petals. Plant Physiol. Biochem. 112, 335-345. doi: 10.1016/j.plaphy.2017.01.019

Li, C., Wu, J., Hu, K. D., Wei, S. W., Sun, H. Y., Hu, L. Y., et al. (2020). PyWRKY26 and PybHLH3 cotargeted the PyMYB114 promoter to regulate anthocyanin biosynthesis and transport in red-skinned pears. Hortic. Res. 7:37. doi: 10.1038/ s41438-020-0254-Z

Li, D., Liu, X., Shu, L., Zhang, H., Zhang, S., Song, Y., et al. (2020). Global analysis of the AP2/ERF gene family in rose (Rosa chinensis) genome unveils the role of RcERF099 in Botrytis resistance. BMC Plant Biol. 20:553. doi: 10.1186/s12870020-02740-6

Li, Y., Shan, X., Zhou, L., Gao, R., Yang, S., Wang, S., et al. (2019). The R2R3MYB factor FhMYB5 from Freesia hybrida contributes to the regulation of anthocyanin and proanthocyanidin biosynthesis. Front. Plant Sci. 9:1935. doi: 10.3389/fpls.2018.01935

Li, Z., Zhao, M., Jin, J., Zhao, L., and Xu, Z. (2018). Anthocyanins and their biosynthetic genes in three novel-colored Rosa rugosa cultivars and their parents. Plant Physiol. Biochem. 129, 421-428. doi: 10.1016/j.plaphy.2018.06. 028

Lim, S. H., Park, B., Kim, D. H., Park, S., Yang, J. H., Jung, J. A., et al. (2020). Cloning and functional characterization of dihydroflavonol 4-reductase gene involved in anthocyanin biosynthesis of chrysanthemum. Int. J. Mol. Sci. 21:7960. doi: 10.3390/ijms21217960

Liu, H., Lou, Q., Ma, J., Su, B., Gao, Z., and Liu, Y. (2019). Cloning and functional characterization of dihydroflavonol 4-Reductase gene involved in anthocyanidin biosynthesis of grape hyacinth. Int. J. Mol. Sci. 20:4743. doi: 10.3390/ijms20194743

Liu, Y., Lin-Wang, K., Espley, R. V., Wang, L., Li, Y., Liu, Z., et al. (2019). StMYB44 negatively regulates anthocyanin biosynthesis at high temperatures in tuber flesh of potato. J. Exp. Bot. 70, 3809-3824. doi: 10.1093/jxb/erz194

Livak, K. J., and Schmittgen, T. D. (2001). Analysis of relative gene expression data using real-time quantitative PCR and the 2- $\Delta \Delta$ CT method. Methods 25, 402-408. doi: 10.1006/meth.2001.1262

Lloyd, A., Brockman, A., Aguirre, L., Campbell, A., Bean, A., Cantero, A., et al. (2017). Advances in the MYB-bHLH-WD repeat (MBW) pigment regulatory model: addition of a WRKY factor and co-option of an anthocyanin MYB for betalain regulation. Plant Cell Physiol. 58, 1431-1441. doi: 10.1093/pcp/pcx075

Lou, Q., Liu, Y., Qi, Y., Jiao, S., Tian, F., Jiang, L., et al. (2014). Transcriptome sequencing and metabolite analysis reveals the role of delphinidin metabolism in flower colour in grape hyacinth. J. Exp. Bot. 65, 3157-3164. doi: 10.1093/jxb/ eru168

Lu, W., Chen, J., Ren, X., Yuan, J., Han, X., Mao, L., et al. (2018). One novel strawberry MADS-box transcription factor FaMADS1a acts as a negative regulator in fruit ripening. Sci. Hortic. 227, 124-131.

Luo, P., Ning, G. G., Wang, Z., Shen, Y. X., Jin, H. A., Li, P. H., et al. (2016). Disequilibrium of flavonol synthase and dihydroflavonol-4-reductase expression associated tightly to White vs. Red color flower formation in plants. Front. Plant Sci 6:1257. doi: 10.3389/fpls.2015.01257 
Ma, R., Xu, S., Zhao, Y. C., Xia, B., and Wang, R. (2016). Selection and validation of appropriate reference genes for quantitative real-time PCR analysis of gene expression in Lycoris aurea. Front. Plant Sci. 7:536. doi: 10.3389/fpls.2016. 00536

Martens, S., Teeri, T., and Forkmann, G. (2002). Heterologous expression of dihydroflavonol 4-reductases from various plants. FEBS Lett. 531, 453-458. doi: 10.1016/s0014-5793(02)03583-4

Mehrtens, F., Kranz, H., Bednarek, P., and Weisshaar, B. (2005). The Arabidopsis transcription factor MYB12 is a flavonol-specific regulator of phenylpropanoid biosynthesis. Plant Physiol. 138, 1083-1096. doi: 10.1104/pp.104.058032

Meyer, P., Heidmann, I., Forkmann, G., and Saedler, H. (1987). A new petunia flower colour generated by transformation of a mutant with a maize gene. Nature 330, 677-678. doi: 10.1038/330677a0

Mortazavi, A., Williams, B. A., Mccue, K., Schaeffer, L., and Wold, B. (2008). Mapping and quantifying mammalian transcriptomes by RNA-Seq. Nat. Methods 5, 621-628. doi: 10.1038/nmeth.1226

Nakatsuka, A., Izumi, Y., and Yamagishi, M. (2003). Spatial and temporal expression of chalcone synthase and dihydroflavonol 4-reductase genes in the Asiatic hybrid lily. Plant Sci. 165, 759-767

Niu, S. S., Xu, C. J., Zhang, W. S., Zhang, B., Li, X., Lin-Wang, K., et al. (2010). Coordinated regulation of anthocyanin biosynthesis in chinese bayberry (Myrica rubra) fruit by an R2R3 MYB transcription factor. Planta 231, 887-899. doi: 10.1007/s00425-009-1095-z

Noda, N., Yoshioka, S., Kishimoto, S., Nakayama, M., Douzono, M., Tanaka, Y., et al. (2017). Generation of blue chrysanthemums by anthocyanin B-ring hydroxylation and glucosylation and its coloration mechanism. Sci. Adv. 3:e1602785. doi: 10.1126/sciadv.1602785

Park, C. H., Yeo, H. J., Kim, Y. J., Nguyen, B. V., Park, Y. E., Sathasivam, R., et al. (2021). Profiles of secondary metabolites (phenolic acids, carotenoids, anthocyanins, and galantamine) and primary metabolites (carbohydrates, amino acids, and organic acids) during flower development in Lycoris radiata. Biomolecules 11:248. doi: 10.3390/biom11020248

Park, C. H., Yeo, H. J., Park, Y. E., Baek, S. A., Kim, J. K., and Park, S. U. (2019). Transcriptome analysis and metabolic profiling of Lycoris radiata. Biology 8:63. doi: 10.3390/biology8030063

Qi, T., Song, S., Ren, Q., Wu, D., Huang, H., Chen, Y., et al. (2011). The Jasmonate-ZIM-domain proteins interact with the WD-Repeat/bHLH/MYB complexes to regulate jasmonate-mediated anthocyanin accumulation and trichome initiation in Arabidopsis thaliana. Plant Cell 23, 1795-1814. doi: 10. 1105/tpc.111.083261

Qi, Y., Gu, C., Wang, X., Gao, S., Li, C., Zhao, C., et al. (2020). Identification of the Eutrema salsugineum EsMYB90 gene important for anthocyanin biosynthesis. BMC Plant Biol. 20:186. doi: 10.1186/s12870-020-02391-7

Quattrocchio, F., Verweij, W., Kroon, A., Spelt, C., Mol, J., and Koes, R. (2006). $\mathrm{PH} 4$ of petunia is an R2R3 MYB protein that activates vacuolar acidification through interactions with basic-helix-loop-helix transcription factors of the anthocyanin pathway. Plant Cell 18, 1274-1291. doi: 10.1105/tpc.105.034041

Rahim, M. A., Busatto, N., and Trainotti, L. (2014). Regulation of anthocyanin biosynthesis in peach fruits. Planta 240, 913-929. doi: 10.1007/s00425-0142078-2

Rameneni, J. J., Choi, S. R., Chhapekar, S. S., Man-Sun, K., Singh, S., Yi, S. Y., et al. (2020). Red Chinese cabbage transcriptome analysis reveals structural genes and multiple transcription factors regulating reddish purple color. Int. J. Mol. Sci. 21:2901. doi: 10.3390/ijms21082901

Rebecca, O. P. S., Boyce, A. N., and Somasundram, C. (2010). Pigment identification and antioxidant properties of red dragon fruit (Hylocereus polyrhizus). Afr. J. Biotechnol. 9, 1450-1454. doi: 10.5897/AJB09.1603

Rice, P., Longden, L., and Bleasby, A. (2000). EMBOSS: the European molecular biology open software suite. Trends Genet. 16, 276-277. doi: 10.1016/S01689525(00)02024-2

Rosas-Saavedra, C., and Stange, C. (2016). Biosynthesis of carotenoids in plants: enzymes and color. Subcell. Biochem. 79, 35-69. doi: 10.1007/978-3-319-391 26-7_2

Saito, K., Yonekura-Sakakibara, K., Nakabayashi, R., Higashi, Y., Yamazaki, M., Tohge, T., et al. (2013). The flavonoid biosynthetic pathway in Arabidopsis: structural and genetic diversity. Plant Physiol. Biochem. 72, 21-34. doi: 10.1016/ j.plaphy.2013.02.001
Sheludko, Y. V., Sindarovska, Y. R., Gerasymenko, I. M., Bannikova, M. A., and Kuchuk, N. V. (2007). Comparison of several Nicotiana species as hosts for high-scale Agrobacterium-mediated transient expression. Biotechnol. Bioeng. 96, 608-614. doi: 10.1002/bit.21075

Shi, Q., Zhou, L., Wang, Y., Li, K., Zheng, B., and Miao, K. (2015). Transcriptomic analysis of Paeonia delavayi wild population flowers to identify differentially expressed genes involved in purple-red and yellow petal pigmentation. PLoS One 10:e0135038. doi: 10.1371/journal.pone.0135038

Shi, S. G., Yang, M., Zhang, M., Wang, P., Kang, Y. X., and Liu, J. J. (2014). Genomewide transcriptome analysis of genes involved in flavonoid biosynthesis between red and white strains of Magnolia sprengeri pamp. BMC Genomics 15:706. doi: 10.1186/1471-2164-15-706

Shimada, N., Sasaki, R., Sato, S., Kaneko, T., Tabata, S., Aoki, T., et al. (2005). A comprehensive analysis of six dihydroflavonol 4-reductases encoded by a gene cluster of the Lotus japonicus genome. J. Exp. Bot. 56, 2573-2585. doi: $10.1093 / \mathrm{jxb} / \mathrm{eri} 251$

Sun, C., Deng, L., Du, M., Zhao, J., Chen, Q., Huang, T., et al. (2020). A transcriptional network promotes anthocyanin biosynthesis in tomato flesh. Mol. Plant 13, 42-58. doi: 10.1016/j.molp.2019.10.010

Suzuki, K., Suzuki, T., Nakatsuka, T., Dohra, H., Yamagishi, M., Matsuyama, K., et al. (2016). RNA-seq-based evaluation of bicolor tepal pigmentation in Asiatic hybrid lilies (Lilium spp.). BMC Genomics 17:611. doi: 10.1186/s12864-0162995-5

Takos, A. M., Jaffé, F. W., Jacob, S. R., Bogs, J., Robinson, S. P., and Walker, A. R. (2006). Light-induced expression of a MYB gene regulates anthocyanin biosynthesis in red apples. Plant Physiol. 142, 1216-1232. doi: 10.1104/pp.106. 088104

Tanaka, Y., Sasaki, N., and Ohmiya, A. (2008). Biosynthesis of plant pigments: anthocyanins, betalains and carotenoids. Plant J. 54, 733-749. doi: 10.1111/j. 1365-313X.2008.03447.x

Tang, Q., Tian, M., An, G., Zhang, W., Chen, J., and Yan, C. (2017). Rapid identification of the purple stem Ps gene of Chinese kale (Brassica oleracea var. alboglabra) in a segregation distortion population by bulked segregant analysis and RNA sequencing. Mol. Breed. 37:153. doi: 10.1007/s11032-017-0752-3

Wang, Q., Wang, Y., Sun, H., Sun, L., and Zhang, L. (2020). Transposon-induced methylation of the RsMYB1 promoter disturbs the anthocyanin accumulation in red-fleshed radish. J. Exp. Bot. 71, 2537-2550. doi: 10.1093/jxb/eraa010

Xiang, L., Liu, X., Li, H., Yin, X., Grierson, D., Li, F., et al. (2019). CmMYB\#7, an R3 MYB transcription factor, acts as a negative regulator of anthocyanin biosynthesis in chrysanthemum. J. Exp. Bot. 70, 3111-3123.

Xie, D. Y., Sharma, S. B., Paiva, N. L., Ferreira, D., and Dixon, R. A. (2003). Role of anthocyanidin reductase, encoded by BANYULS in plant flavonoid biosynthesis. Science 299, 396-399. doi: 10.1126/science.1078540

Xie, Y., Tan, H., Ma, Z., and Huang, J. (2016). DELLA proteins promote anthocyanin biosynthesis via sequestering MYBL2 and JAZ suppressors of the MYB/bHLH/WD40 complex in Arabidopsis thaliana. Mol. Plant 9, 711-721. doi: 10.1016/j.molp.2016.01.014

Xu, L., Yang, P., Feng, Y., Xu, H., Cao, Y., Tang, Y., et al. (2017). Spatiotemporal transcriptome analysis provides insights into bicolor tepal development in Lilium “Tiny Padhye”. Front. Plant Sci. 8:398. doi: 10.3389/fpls.2017.00398

Xu, W., Dubos, C., and Lepiniec, L. (2015). Transcriptional control of flavonoid biosynthesis by MYB-bHLH-WDR complexes. Trends Plant Sci. 20, 176-185. doi: 10.1016/j.tplants.2014.12.001

Yamagishi, M., Toda, S., and Tasaki, K. (2014). The novel allele of the LhMYB12 gene is involved in splatter-type spot formation on the flower tepals of Asiatic hybrid lilies (Lilium spp.). New Phytol. 201, 1009-1020. doi: 10.1111/nph.12 572

Yang, X., Xia, X., Zhang, Z., Nong, B., Zeng, Y., Wu, Y., et al. (2019). Identification of anthocyanin biosynthesis genes in rice pericarp using PCAMP. Plant Biotechnol. J. 17, 1700-1702. doi: 10.1111/pbi.13133

Young, M. D., Wakefield, M. J., Smyth, G. K., and Oshlack, A. (2010). Gene ontology analysis for RNA-seq: accounting for selection bias. Genome Biol. 11:R14. doi: 10.1186/gb-2010-11-2-r14

Yue, Y., Liu, J., Shi, T., Chen, M., Li, Y., Du, J., et al. (2019). Integrating transcriptomic and GC-MS metabolomic analysis to characterize color and aroma formation during tepal development in Lycoris longituba. Plants 8:53. doi: $10.3390 /$ plants 8030053 
Zhai, R., Wang, Z., Yang, C., Lin-Wang, K., Espley, R., Liu, J., et al. (2019). PbGA2ox8 induces vascular-related anthocyanin accumulation and contributes to red stripe formation on pear fruit. Hortic. Res. 6:137. doi: 10.1038/s41438019-0220-9

Zhang, F., Wang, T., Shu, X., Wang, N., Zhuang, W., and Wang, Z. (2020). Complete chloroplast genomes and comparative analyses of L. chinensis, L. anhuiensis, and L. aurea (Amaryllidaceae). Int. J. Mol. Sci. 21:5729. doi: 10.3390/ijms21165729

Zhang, H., Jin, J., Tang, L., Zhao, Y., Gu, X., Gao, G., et al. (2011). PlantTFDB 2.0: update and improvement of the comprehensive plant transcription factor database. Nucleic Acids Res. 9, D1114-D1117. doi: 10.1093/nar/gkq1141

Zhang, X., Xu, Z., Yu, X., Zhao, L., Zhao, M., Han, X., et al. (2019). Identification of two novel R2R3-MYB transcription factors, PsMYB114L and PsMYB12L, related to anthocyanin biosynthesis in Paeonia suffruticosa. Int. J. Mol. Sci. 20:1055. doi: 10.3390/ijms20051055

Zhang, Y., Butelli, E., and Martin, C. (2014). Engineering anthocyanin biosynthesis in plants. Curr. Opin. Plant Biol. 19, 81-90. doi: 10.1016/j.pbi.2014.05.011

Zhao, D., Jiang, Y., Ning, C., Meng, J., Lin, S., Ding, W., et al. (2014). Transcriptome sequencing of a chimaera reveals coordinated expression of anthocyanin biosynthetic genes mediating yellow formation in herbaceous peony (Paeonia lactiflora Pall). BMC Genomics 15:689. doi: 10.1186/1471-2164-15-689

Zhao, D., and Tao, J. (2015). Recent advances on the development and regulation of flower color in ornamental plants. Front. Plant Sci. 6:261. doi: 10.3389/fpls. 2015.00261

Zhao, D., Tao, J., Han, C., and Ge, J. (2012). Flower color diversity revealed by differential expression of flavonoid biosynthetic genes and flavonoid accumulation in herbaceous peony (Paeonia lactiflora Pall.). Mol. Biol. Rep. 39, 11263-11275. doi: 10.1007/s11033-012-2036-7

Zhong, C., Tang, Y., Pang, B., Li, X., Yang, Y., Deng, J., et al. (2020). The R2R3-MYB transcription factor GhMYB1a regulates flavonol and anthocyanin accumulation in Gerbera hybrida. Hortic. Res. 7:78. doi: 10.1038/s41438-0200296-2
Zhou, C., Mei, X., Rothenberg, D. O'., Yang, Z., Zhang, W., Wan, S., et al. (2020). Metabolome and transcriptome analysis reveals putative genes involved in anthocyanin accumulation and coloration in white and pink tea (Camellia sinensis) Flower. Molecules 25:190. doi: 10.3390/molecules2501 0190

Zhou, H., Lin-Wang, K., Wang, H., Gu, C., Dare, A. P., Espley, R. V., et al. (2015). Molecular genetics of blood-fleshed peach reveals activation of anthocyanin biosynthesis by NAC transcription factors. Plant J. 82, 105-121. doi: 10.1111/ tpj. 12792

Zhu, H. H., Yang, J. X., Xiao, C. H., Mao, T. Y., Zhang, J., and Zhang, H. Y. (2019). Differences in flavonoid pathway metabolites and transcripts affect yellow petal colouration in the aquatic plant Nelumbo nucifera. BMC Plant Biol. 19:277. doi: 10.1186/s12870-0191886-8

Conflict of Interest: The authors declare that the research was conducted in the absence of any commercial or financial relationships that could be construed as a potential conflict of interest.

Publisher's Note: All claims expressed in this article are solely those of the authors and do not necessarily represent those of their affiliated organizations, or those of the publisher, the editors and the reviewers. Any product that may be evaluated in this article, or claim that may be made by its manufacturer, is not guaranteed or endorsed by the publisher.

Copyright (c) 2021 Wang, Shu, Zhang, Zhuang, Wang and Wang. This is an openaccess article distributed under the terms of the Creative Commons Attribution License (CC BY). The use, distribution or reproduction in other forums is permitted, provided the original author(s) and the copyright owner(s) are credited and that the original publication in this journal is cited, in accordance with accepted academic practice. No use, distribution or reproduction is permitted which does not comply with these terms 